\title{
Thermosensitive nanofibers loaded with ciprofloxacin as antibacterial wound dressing materials
}

Heyu Li ${ }^{1}$, Gareth R. Williams ${ }^{2}$, Junzi Wu ${ }^{1}$, Yao $\mathrm{Lv}^{1}$, Xiaozhu Sun ${ }^{1}$, Huanling $\mathrm{Wu}^{1}$,

Li-Min $\mathrm{Zhu}^{1 *}$

1. College of Chemistry, Chemical Engineering and Biotechnology, Donghua University, Shanghai, 201620, China

2. UCL School of Pharmacy, 29-39 Brunswick Square, London, WC1N 1AX, UK Corresponding author E-mail: 1zhu@dhu.edu.cn Tel: 00862167792655

\section{Abstract}

To obtain wound dressings which could be removed easily without secondary injuries, we prepared thermoresponsive electrospun fiber mats containing poly(di(ethylene glycol) methyl ether methacrylate (PDEGMA). Blend fibers of PDEGMA and (poly(1-lactic acid-co-e-caprolactone) (P(LLA-CL) were fabricated via electrospinning,

15 and analogous fibers containing the antibiotic ciprofloxacin (CIF) were also prepared. Smooth cylindrical fibers were obtained, albeit with a small amount of beading visible for the ciprofloxacin-loaded fibers. X-ray diffraction showed the drug to exist in the amorphous physical form post-electrospinning. The composite fibers showed distinct thermosensitive properties and gave sustained release of CIF over more than 160

20 hours in vitro. The fibers could promote the proliferation of fibroblasts, and by varying the temperature cells could easily be attached to and detached from the fibers. Antibacterial tests demonstrated that fibers loaded with ciprofloxacin were effective in inhibiting the growth of E. coli and S. aureus. In vivo investigations on rats indicated that the composite PDEGMA/P(LLA-CL) fibers loaded with CIF had much more potent wound healing properties than a commercial gauze and CIF-loaded fibers made solely of P(LLA-CL). These results demonstrate the potential of PDEGMA/P(LLA-CL)/ ciprofloxacin fibers as advanced wound dressing materials. 
Keywords: Thermosensitive, PDEGMA, P(LLA-CL), Ciprofloxacin, Electrospinning,

\section{Introduction}

Wound dressings comprise an important segment of the wound care market (Boateng et al., 2008). Contemporary dressings are designed to maintain a suitable environment for healing: to do this, they must absorb excess exudates; offer thermal insulation, gaseous and fluid exchanges; and, avoid allergic reactions, toxicity, scarring and sensitization. They must also be sterile (Zahedi et al., 2010). A range of dressing types has been explored, including films, foams, hydrogels, hydrocolloids and hydrofibers (Thomas et al., 1988; Cho et al., 2002; Newman et al., 2006; Thu et al., 2012; Tan et al., 2013).

Nanofiber-based dressings made by electrospinning have several clear advantages as wound dressing materials. In addition to their high surface area-to-volume ratio, porosity and the great tunability of these systems, they also often resemble the morphological structure of the extra-cellular matrix (ECM) and have good biocompatibility (Charernsriwilaiwat et al., 2012; Pelipenko et al., 2013; Thakur et al., 2008). It has been found that electrospun wound dressings have good hemostasis, absorbability, and semi-permeability (Li et al., 2016; Zhang et al., 2005). Multiple therapeutic compounds can easily be incorporated into nanofibers via this route, leading to facile production of multifunctional dressings. In the clinic, wound dressings need to be replaced periodically, which inevitably causes some secondary injury as tissue adhered to the dressing is pulled off during replacement. This compromises the healing process. This is potentially a particular problem with nanofiber-based dressings because of their biomimetic structures. To solve this

55 problem, thermosensitive polymers can be employed.

Thermoresponsive polymers undergo phase transitions in response to temperature 
changes, and have attracted widespread attention (Dimitrov et al., 2007; Behl et al., 2010). Some researchers have demonstrated that thermosensitive materials can effectively control cell adhesion and detachment through variation of the temperature (Schild, 1992). Poly(N-isopropylacrylamide) (PNIPAM), with a lower critical solution temperature (LCST) of $32{ }^{\circ} \mathrm{C}$, has been by far the most studied thermoresponsive polymer (Akiyama et al., 2004; Gil and Hudson, 2007), and thermosensitive would dressings made from PNIPAM have been reported in the literature (Yang et al., 2008; Wang et al., 2006; Heilmann et al., 2008; Reddy et al., 2008). Such systems exhibit a chain-to-globule transition as the temperature is raised through the LCST. The strength of interactions between the polymer and cells is generally much greater above the LCST (Akiyama et al., 2004), and thus if the LCST is a little below the human body temperature a low-temperature treatment could be applied to reduce attractions between the polymer and cells. This will promote detachment of cells from the dressing and thereby can reduce secondary injuries, as the dressing is less likely to bring tissue with it upon changing. As a result pain and secondary injury can be much reduced. There are several reports of thermosensitive wound dressings in the literature. For example, chitosan containing polyurethane/PNIPAM polymer membranes have shown good antibacterial ability

75 against $P$. aeruginosa and $S$. aureus. They also could support the growth of 3T3fibroblasts (Yang et al., 2008). Wang et al. reported that polymer membranes synthesized from $\mathrm{N}$-isopropylacrylamide, methyl methacrylate, and 2-hydroxyethyl methacrylate exhibit excellent thermosensitivity and cell attachment/detachment properties. The highest detachment efficiency with L929 cells was about $90 \%$ at $15{ }^{\circ} \mathrm{C}$ (Wang et al., 2006). Most of the thermosensitive dressings based on PNIPAM were not made by electrospinning and do not have the advantages of nanofibers, however.

In recent years, different oligo(ethylene glycol) methyl ether methacrylate (OEGMA)-based polymers have been synthesized and studied as alternatives to 85 PNIPAM. The former have graft structures composed of a carbon-carbon backbone 
and multiple oligo(ethylene glycol) side-chains (Lutz, et al., 2008). They have inherent advantages over PNIPAM, including better bio-repellency below the LCST, reversible phase transitions without marked hysteresis, and bio-inert properties (Lutz, 2011).

90 In this work we used di(ethylene glycol) methyl ether methacrylate (DEGMA) to synthesize the thermosensitive polymer PDEGMA, which has an LCST just below $30{ }^{\circ} \mathrm{C}$ (Han et al., 2003; Becer et al., 2008; Ishizone et al., 2008). To produce high-quality fibers with regular and homogenous morphologies, PDEGMA was then electrospun into nanofibers in combination with P(LLA-CL). The latter has been widely utilized in electrospinning, and found to have high spinnability as well as good biocompatibility, biodegradability and mechanical properties (Li et al., 2009). These are all necessary for wound dressing materials. Ciprofloxacin, a fluoroquinolone antibiotic, was additionally loaded in the composite nanofibers. In vitro and in vivo studies of the drug-loaded PDEGMA/ P(LLA-CL) nanofibers were undertaken.

\section{Materials and methods}

\subsection{Materials}

Di(ethylene glycol) methyl ether methacrylate (DEGMA, 95\%),

$1051,1^{\prime}$-azobis(cyclohexanecarbonitrile) (VAZO-88, 98\%), ciprofloxacin (CIF, $\geq 98 \%$ ), phosphate-buffered saline (PBS), sodium azide, penicillin, trypsin and thiazolyl blue (MTT) were purchased from Sigma-Aldrich Ltd (USA). Dimethyl formamide (DMF), dichloromethane, formaldehyde and n-hexane were obtained from the Sinopharm Chemical Reagent Co., Ltd (China). A copolymer of P(LLA-CL) (50:50; Mw = 34.5 $110 \times 10^{4} \mathrm{~g} / \mathrm{mol}$ ) was provided by Nara Medical University (Japan). 1,1,1,3,3,3-hexafluoro-2-propanol (HFIP, 99.5\%) was procured from the Aladdin Industrial Corporation (China). CD34 and collagen type I polyclonal antibodies, $\beta$-actin monoclonal antibody, PVDF touch, and ECL luminescence kits were obtained from Santa Cruz (USA), and the corresponding secondary antibodies were purchased 
115 from the Zhongshan Golden Bridge Company (China). L929 cells were provided by the Institute of Biochemistry and Cell Biology (Chinese Academy of Sciences, China). Dimethyl sulfoxide (DMSO) and DMEM culture medium were sourced from Jinuo Biological Medicine Technology Ltd. (China). All other chemicals used were analytical grade, and water was doubly distilled before use.

\subsection{Thermosensitive polymer synthesis and characterization}

The PDEGMA polymer was prepared by free-radical polymerization according to the literature (Sun et al., 2014). Briefly, $3.76 \mathrm{~g}$ of DEGMA and $0.005 \mathrm{~g}$ of the initiator VAZO-88 were dissolved in $4 \mathrm{~mL}$ of DMF, to give a molar ratio of DEGMA : VAZO-88 of 2000:1. The reaction mixture was heated at $90{ }^{\circ} \mathrm{C}$ under nitrogen for 1.5 h. The resultant solid was initially dissolved in dichloromethane before PDEGMA was precipitated through the addition of n-hexane. The dissolution/precipitation process was repeated three times and then the product dried for 3 days in a vacuum oven (DZF-6050, Shanghai Laboratory Instrument Work Co. Ltd., China). Successful 130 polymerization was verified by ${ }^{1} \mathrm{H}$ nuclear magnetic resonance (AV-400 instrument, Bruker, Germany). Molecular weights ( $\mathrm{Mw}$ and $\mathrm{Mn}$ ) and molecular weight distributions were determined by gel permeation chromatography (GPC) measurements on a Waters LS measurement system (Waters, USA) with tetrahydrofuran (THF) as the solvent. The flow rate was $1.0 \mathrm{~mL} / \mathrm{min}$, and the column

135 temperature was $35{ }^{\circ} \mathrm{C}$. The molecular weight distribution of PDEGMA was calibrated with standard polystyrene samples.

\subsection{Preparation of electrospinning solutions}

PDEGMA and P(LLA-CL) were dissolved in HFIP under magnetic stirring for $10 \mathrm{~h}$ at

140 room temperature, resulting in clear and homogenous solutions. The component ratio of PDEGMA to P(LLA-CL) was 1:1, 1:2 or 1:3 (w/w), and the total concentration of polymer was $9 \%(\mathrm{w} / \mathrm{v})$. Solutions containing P(LLA-CL) alone were also prepared as controls. CIF was added into certain solutions at a drug to polymer ratio of 1:10 (w/w). Full details of all solutions are listed in Table 1. 
Table 1. Details of the spinning solutions used in this work. All solutions were prepared in HFIP

\begin{tabular}{|c|c|c|c|}
\hline Sample & Solution contents & $\begin{array}{c}\text { PDEGMA to } \\
\text { P(LLA-CL) } \\
\text { ratio }(w / w)\end{array}$ & $\begin{array}{c}\text { Drug } \\
\text { concentration } \\
(\% \mathrm{w} / \mathrm{v})\end{array}$ \\
\hline S1 & PDEGMA/ P(LLA-CL) & $1: 1$ & --- \\
\hline S2 & PDEGMA/ P(LLA-CL) & $1: 2$ & --- \\
\hline S3 & PDEGMA/ P(LLA-CL) & $1: 3$ & --- \\
\hline $\mathrm{S} 4$ & P(LLA-CL) & --- & --- \\
\hline S5 & $\begin{array}{c}\text { PDEGMA/ P(LLA-CL) } \\
\text { /CIF }\end{array}$ & $1: 1$ & 0.9 \\
\hline S6 & $\begin{array}{c}\text { PDEGMA/ P(LLA-CL) } \\
\text { /CIF }\end{array}$ & $1: 2$ & 0.9 \\
\hline S7 & $\begin{array}{c}\text { PDEGMA/ P(LLA-CL) } \\
\text { /CIF }\end{array}$ & $1: 3$ & 0.9 \\
\hline S8 & $\mathrm{P}(\mathrm{LLA}-\mathrm{CL}) / \mathrm{CIF}$ & --- & 0.9 \\
\hline
\end{tabular}

\subsection{Preparation of fibers}

The solution required for electrospinning was placed into a $5 \mathrm{~mL}$ plastic syringe fitted with a stainless steel needle (internal diameter $0.5 \mathrm{~mm}$ ) and the syringe mounted on a syringe pump (KDS100, Cole-Parmer, USA). The solution was expelled from the syringe at a rate of $1.0 \mathrm{~mL} / \mathrm{h}$ and a high voltage power supply (ZGF-2000, Shanghai

155 Sute Electrical Co. Ltd., China) used to apply a voltage of $16 \mathrm{kV}$ between the needle and a grounded collector (a flat piece of aluminum foil of $10 \times 10 \mathrm{~cm}$ ). The distance between the needle tip and the grounded collector was fixed at $15 \mathrm{~cm}$. The relative humidity was ca. $40 \%$, and the temperature $25^{\circ} \mathrm{C}$. After electrospinning for 8 hours, the products were stored in a vacuum oven at room temperature for $24 \mathrm{~h}$ to remove 


\subsection{Fiber characterization}

To observe the morphology of the fibers, samples were first gold sputter-coated for 60 s under argon to make them electrically conductive. Their morphological structure was then investigated using a scanning electron microscope (SEM; JSM-5600 LV microscope, JEOL, Japan) at a voltage of $10 \mathrm{kV}$. The average fiber diameter for each sample was calculated by the analysis of approximately 100 fibers in SEM images, using the ImageJ software (National Institutes of Health, USA). X-ray diffraction (XRD) patterns were obtained on a D/Max-BR diffractometer (Rigaku, Japan). The instrument is supplied with $\mathrm{Cu} \mathrm{K} \alpha$ radiation $(40 \mathrm{kV} / 30 \mathrm{~mA})$, and patterns were collected over the $2 \theta$ range $5-60^{\circ}$. Fourier transformed infrared spectroscopy (FTIR) was undertaken using a Nicolet-Nexus 670 FTIR spectrometer (Nicolet Instrument Corporation, USA) over the scanning range $500-4000 \mathrm{~cm}^{-1}$ at a resolution of $2 \mathrm{~cm}^{-1}$. The water contact angle (CA) was determined on a contact angle analyzer (DSA 30,

175 Krüss GmbH, Hamburg, Germany) in air. A water droplet (ca. $1 \mu \mathrm{L}$ ) was placed onto the surface of the fibers and the CA recorded. The measurement temperature was varied from $20{ }^{\circ} \mathrm{C}$ to $45^{\circ} \mathrm{C}$ using a heating platform (XMTD-204, JTHF Company, Jintan, China). Five measurements were recorded for each sample, and the results are reported as mean \pm S.D.

180 The water uptake capacity of the fibers and a commercial gauze (as control) was examined in PBS ( $\left.\mathrm{pH} 7.4 ; 37^{\circ} \mathrm{C}\right)$. Experiments were carried out in a humidified incubator, and the samples weighed after $24 \mathrm{~h}$. The water uptake capacity was calculated as follows (Rath et al., 2016):

Water uptake capacity $=\left(\mathrm{W}-\mathrm{W}_{0}\right) / \mathrm{W}_{0} \times 100 \%$

185 where $\mathrm{W}$ represents the weight of the sample after immersion in water and $\mathrm{W}_{0}$ the weight of the dry sample.

\subsection{In vitro drug release}

The loading of CIF in the nanofibers was first determined before the release 
experiment. $100 \mathrm{mg}$ of fibers was dissolved in $10 \mathrm{~mL}$ of HFIP. The amount of dissolved CIF was then determined using a UV spectrophotometer (UV-1800, SHJH Company, China) at $269 \mathrm{~nm}$. The amount of CIF present in the nanofibers was back-calculated against a predetermined calibration curve. The results are reported as mean \pm S.D. from three independent determinations.

195 Drug release experiments were then conducted at $37^{\circ} \mathrm{C}$ and $110 \mathrm{rpm}$ in a thermostatic shaking incubator (Jintan Instrument Co. Ltd., China). $100 \mathrm{mg}$ of each fiber mat was separately immersed in $30 \mathrm{~mL}$ of a release medium ( $\mathrm{pH} 7.4 \mathrm{PBS}$ ). At predetermined time points, $1 \mathrm{~mL}$ of the test medium was withdrawn and an equal amount of fresh preheated PBS was added. The amount of CIF released was determined using UV spectroscopy. Experiments were carried out in triplicate and the results are reported as mean \pm S.D.

\subsection{Cell growth}

Fibers were electrospun directly onto cover slips for these experiments 20 cover slips were placed onto the collector plate and $5 \mathrm{~mL}$ of spinning solution was dispensed onto them. The slips were subsequently placed in the wells of 24-well plates, with untreated cover slips used as a negative control. Before cell culture, the plates were sterilized with alcohol steam for 24 h. $400 \mu \mathrm{L}$ of dissociated L929 fibroblasts $(1.0 \times$ $10^{4}$ cells/mL, in DMEM supplemented with $10 \%$ v/v FBS and $1 \%$ (v/v)

210 penicillin-streptomycin) was added to each well, and the plate placed in an incubator $\left(37^{\circ} \mathrm{C}, 5 \% \mathrm{CO}_{2}\right)$. After 1,3 , or 5 days, the culture medium in each well was replaced by $360 \mu \mathrm{L}$ of fresh DMEM and $40 \mu \mathrm{L}$ of an MTT solution $(5 \mathrm{mg} / \mathrm{mL}$ thiazolyl blue in PBS). The plate was then incubated for $6 \mathrm{~h}$, after which $400 \mu \mathrm{L}$ DMSO was added to each well and the plates shaken for $30 \mathrm{~min}$ at room temperature. The purple solution

215 in each well was transferred to a 96-well plate and the number of cells assessed via the OD values at $570 \mathrm{~nm}$. These were recorded using a microplate reader (Multiskan, ThermoFisher, USA). Cell viability with a $0.9 \%$ CIF solution was also explored (see Supporting Information for full details). 
To test the detachment behavior of the thermosensitive nanofibers, L929 fibroblasts were seeded onto cover slips covered with fibers in the 24 -well plates as described above, with fiber-free slips as a control. After incubation $\left(37^{\circ} \mathrm{C}, 5 \% \mathrm{CO}_{2}\right)$ for two days, cell growth had exceeded $80 \%$ confluence. The samples were then transferred

225 to an incubator at $25{ }^{\circ} \mathrm{C}$ for $30 \mathrm{~min}$. Subsequently, the contents of each well were aspirated and transferred to a fresh 6-well plate, which was returned to the $37{ }^{\circ} \mathrm{C}$ incubator to allow the detached cells to reattach to the new plates and resume growth (Wang et al., 2006). The reattached cells were studied using an inverted microscope (Olympus IX71, Japan). The number of cells remaining in each well of the original 230 plates was assessed using the MTT assay as detailed previously. For each fiber sample, cell detachment assays were performed three times, and the results are expressed as mean \pm S.D. Controls were established using cell-seeded slips incubated at $37^{\circ} \mathrm{C}$ for the same period of time, but with no $25^{\circ} \mathrm{C}$ treatment. The detachment ration was calculated as follows:

235 Detachment ratio $=(\mathrm{A}-\mathrm{B}) / \mathrm{A} \times 100 \%$

where $\mathrm{A}$ is the number of cells in the samples without the $25^{\circ} \mathrm{C}$ treatment, and $\mathrm{B}$ is the number of cells remaining after this treatment.

\subsection{Antibacterial activity}

240 Antibacterial activities of the nanofibers were studied using disc diffusion method of the US Clinical and Laboratory Standards Institute (CLSI) (Unnithan et al., 2012). In the assessment, $10^{5}$ colony forming units (CFUs) of E. coli (Gram-negative; ATCC 25922) and S. aureus (Gram-positive; ATCC 27853) were selected as representative microorganisms, and cultured on lauria broth (LB) and nutrient broth (NB) agar plates

245 respectively. The fiber samples were cut into circular discs $2 \mathrm{~cm}$ in diameter and sterilized by $75 \%$ alcohol steam for $24 \mathrm{~h}$. $100 \mu \mathrm{L}$ of microorganism solution was cultured on an agar plate in a petri dish and then each of the samples was placed on the surface of the agar plate. Next, all the dishes were incubated at $37{ }^{\circ} \mathrm{C}$ for $72 \mathrm{~h}$. Inhibition zones were measured after incubation for 24 and $72 \mathrm{~h}$. The antibacterial 
property of a control CIF solution was also investigated using a shake flask method, as detailed in the Supporting Information.

\subsection{In vivo experiments}

All animal experiments were performed under certificate SYZK 2014-0022 issued by the Shanghai Science and Technology Committee, in full accordance with their rules and regulations. Male SD (Sprague Dawley) rats from SLAC Laboratory Animal Inc. (China) weighing from 220-250 g were used as animal models. The animals were anesthetized with an intraperitoneal injection of pentobarbitone sodium $(50 \mathrm{mg} / \mathrm{kg})$ and then a $3 \mathrm{~cm} \times 3 \mathrm{~cm}\left(900 \mathrm{~mm}^{2}\right)$ open excision type wound was created to the depth of loose subcutaneous tissue on their upper backs. S6 or S8 fiber mats (see Table 1; $3 \mathrm{~cm} \times 3 \mathrm{~cm}\left[900 \mathrm{~mm}^{2}, 0.1 \mathrm{~g}\right]$ ) sterilized by alcohol steam for $24 \mathrm{~h}$ were used to cover the skin wounds. An antiseptic gauze loaded with ampicillin (Shanghai Yinjing Medical Supplies Company Ltd, China) was placed over each of the mats, and the gauze edge was sutured to the skin around the wound area. This is in 265 accordance with previously reported protocols for such studies (Yang et al., 2011). Wounds i) sealed with ampicillin-loaded gauzes; ii) sealed with the same gauze soaked with a $0.9 \% \mathrm{w} / \mathrm{v}$ CIF solution; and, iii) sprinkled with a $0.9 \% \mathrm{w} / \mathrm{v}$ CIF solution $(1.1 \mathrm{ml})$ were used as control groups. There were seven rats in each group.

$270 \quad 2.11$ Macroscopic observation of the wound healing process

After recovery from anaesthesia, the rats were housed individually in disinfected cages at a temperature of $25 \pm 2{ }^{\circ} \mathrm{C}$. The dressings in all the groups were replaced every three days. The rats in CIF solution group were treated with a $0.9 \% \mathrm{CIF}$ solution every three days also. Before dressing replacement, the fibers and gauzes on

275 the wounds were treated by saline at $25^{\circ} \mathrm{C}$. Replacement of the dressings every three days was undertaken to meet local clinical requirements in observing the healing process and avoid infection. On day 0, 5, 14 and 21 post-operation, the appearance of the wound was photographed. The area of unhealed wound was measured from the image using the Photoshop software (Adobe, USA). The relative wound area was 
determined from the following equation:

Relative wound area $=\mathrm{A}_{\mathrm{t}} / \mathrm{A}_{0} \times 100 \%$

where $A_{t}$ and $A_{0}$ are the wound areas on the specified day and operation day, respectively.

2852.12 Histological, immunohistochemical and toxicological examinations

21 days after operation, three rats from each group were sacrificed using a large dose of anesthetic. The wound sections were excised, collected and cut into small sections $(4 \mu \mathrm{m})$. The wound sections were fixed with $10 \%$ formaldehyde, rinsed in water and placed in a transparent tissue block of melted paraffin, ensuring that the samples were completely immersed in the block. Sections were then block fixed on a slicer for hematoxylin-eosin (HE) staining. For staining, the sections were washed with distilled water, stained with hematoxylin, rinsed under running tap water, and differentiated with $0.3 \%$ acid alcohol. They were next rinsed under running tap water again, stained with eosin for $2 \mathrm{~min}$, then dried, cleaned and mounted. HE-stained sections were studied with a light microscope (Nikon Eclipse E400, Japan).

In addition, some sections of tissue in the wound area from the same rats were homogenized in RIPA lysis buffer. The total tissue protein was collected and quantified by BCA protein kits. $60 \mu \mathrm{g}$ of each sample was processed by SDS-PAGE electrophoresis on a $10 \%$ SDS-PAGE gel at $100 \mathrm{~V}$, followed by transfer to a PVDF membrane (Millipore Corp., USA). After blocking with $5 \%$ (w/v) skimmed milk for $2 \mathrm{~h}$ at room temperature, the membrane was washed and incubated with primary antibody at a dilution of 1:1000 for $30 \mathrm{~min}$ at room temperature, and then stored at 4 ${ }^{\circ} \mathrm{C}$ overnight. The membranes were then washed three times with PBS containing $0.05 \%$ Tween 20 and reacted with a secondary HRP-conjugated antibody for $1 \mathrm{~h}$.

305 After being washed three times with PBS containing $0.05 \%$ Tween 20 and reacted with the ECL luminescent reagent, the membranes were exposed and scanned. The expression of CD34 and collagen type I in the regenerated tissues was detected with $\beta$-actin as a protein loading control.

To probe skin irritation, the area around the wound was studied for any kind of 
310 oedema or erythema on the skin during the period of healing (Rath et al., 2016). To further examine the toxicity of the fibers, blood from rats in the fiber groups (S6 and S8) was collected. This was assayed for a range of markers including the red blood cell (RBC), white blood cell (WBC), and platelet counts. Hemoglobin, serum protein, serum creatinine, hematocrit, serum glutathione, total cholesterol, uric acid, aspartate 315 amino transferase (AST) and glutamine transaminase (ALT) were also quantified using an automated Olympus AU5400 biochemistry analyzer (Olympus, Japan) to Measurements obtained from normal rats without any wounds or treatment were used as the control group.

\section{Results and discussion.}

\subsection{Synthesis and characterization of PDEGMA}

The successful formation of PDEGMA was verified by ${ }^{1} \mathrm{H}$ NMR (Figure 1). The spectrum of DEGMA (Figure 1(a); $\mathrm{D}_{2} \mathrm{O}, 400 \mathrm{MHz}$ ) shows resonances as follows: $\delta$ (ppm): $6.00\left(1 \mathrm{H}, \mathrm{CH}_{2}=\mathrm{C}\right), 5.57\left(1 \mathrm{H}, \mathrm{CH}_{2}=\mathrm{C}\right), 4.18\left(2 \mathrm{H},-\mathrm{CH}_{2^{-}}\right), 3.67\left(2 \mathrm{H},-\mathrm{CH}_{2^{-}}\right)$, $3.57\left(2 \mathrm{H},-\mathrm{CH}_{2}-\right), 3.47\left(2 \mathrm{H},-\mathrm{CH}_{2}-\right), 3.22\left(3 \mathrm{H},-\mathrm{OCH}_{3}\right), 1.78\left(3 \mathrm{H}, \mathrm{CH}_{2}=\mathrm{C}^{-} \mathrm{CH}_{3}\right)$. The spectrum of the polymer PDEGMA, shown in Figure 1(b), reveals the absence of vinyl groups. The signals at $\delta$ of 6.0 and 5.57 of DEGMA have disappeared, demonstrating that PDEGMA was successfully obtained. The molecular weights (Mw and $\mathrm{Mn}$ ) and molecular weight distributions of PEDGMA determined by GPC were $35,573,29,734$, and 1.19 , respectively.

\section{Figure 1}

\subsection{Fiber morphology}

335 Scanning electron microscopy (SEM) images of the electrospun materials are shown in Figure 2, together with their diameter distributions. Fibers were successfully prepared from all the solutions studied. Considering the fibers without CIF, the average diameter can be seen to vary from $948 \pm 132 \mathrm{~nm}(\mathrm{~S} 4)$ to $476 \pm 189 \mathrm{~nm}$ (S1) 
as increasing amounts of PDEGMA are incorporated into the nanofibers. This is

expected to be related to the rise in conductivity of the electrospinning solutions with increasing PDEGMA content (Yin et al., 2013). The diameter distributions of fibers composed of P(LLA-CL) alone (S4) are also observed to be more uniform than those of the blend systems.

The trends in fiber diameter and diameter distributions in the fibers loaded with

345 CIF are similar to those without drugs. However, the diameters of the CIF loaded fibers are rather narrower. For instance, the average diameter of S2 is $619 \mathrm{~nm}$ as compared to $354 \mathrm{~nm}$ for S6. This may result from an increase in conductivity following the addition of CIF; this is known to facilitate elongation of the polymer jet and to generate narrower fibers (Su et al., 2009). There is also evidence for some

350 beading in the CIF loaded fibers depicted in Figure 2 (S5-S8), which might result from the addition of CIF causing increased viscosity (Pham et al., 2006).

\section{Figure 2}

3.3. X-ray diffraction

X-ray diffraction (XRD) patterns are presented in Figure 3. Ciprofloxacin (CIF) shows a number of well-defined Bragg reflections in its diffraction pattern, demonstrating its crystalline nature. However, all the fibers loaded with CIF display only two broad diffuse features centered at around $7^{\circ}$ and $17^{\circ} 2 \theta$, indicating they are amorphous materials. These results demonstrate that CIF was converted into the amorphous physical form by the electrospinning process.

\section{Figure 3}

\subsection{Thermoresponsive behavior}

The thermosensitive behavior of the nanofibers loaded with CIF was studied, and the results are depicted in Figure 4(a). The neat P(LLA-CL) fibers loaded with CIF are hydrophobic regardless of the temperature, with perhaps very slightly lower contact 
angles at lower temperatures. In contrast, as the temperature is reduced from $45^{\circ} \mathrm{C}$ to $20^{\circ} \mathrm{C}$, the surfaces of the PDEGMA-containing fibers change significantly, although to different extents. The S5 nanofibers, which contain the largest proportion of PDEGMA (1:1 PDEGMA: P(LLA-CL) w/w), have a contact angle of around $90^{\circ}$ at $45^{\circ} \mathrm{C}$. Below $30{ }^{\circ} \mathrm{C}$, this value decreases dramatically and reaches $0^{\circ}$ at $20{ }^{\circ} \mathrm{C}$. In comparison, the contact angle of the sample with the lowest proportion of PDEGMA

$375(\mathrm{~S} 7,1: 3)$ starts with a contact angle of more than $120^{\circ}$ at $45^{\circ} \mathrm{C}$, which declines to about $90^{\circ}$ at $20^{\circ} \mathrm{C}$. The S6 fibers (1:2) have the most marked thermosensitive behavior, with a decline in contact angles from hydrophobic (ca. $120^{\circ}$ ) at $45^{\circ} \mathrm{C}$ to hydrophilic $\left(15^{\circ}\right)$ at $20^{\circ} \mathrm{C}$.

The contact angles of all the fibers containing PDEGMA thus exhibit a dramatic 380 change near $30^{\circ} \mathrm{C}$, the LCST of PDEGMA. PDEGMA will be soluble in water below the LCST but precipitate at temperatures above it. Below the LCST, the balance between favorable polymer-water interactions and unfavorable hydrophobic interactions lies in favor of the former, allowing solubilization. Above the LCST, this balance is disrupted and polymer-polymer interactions are thermodynamically

385 favored over polymer-water interactions (Lutz et al., 2007; Maeda et al., 2007; Lutz, 2008). As a result, the polymers become hydrophobic above the LCST, explaining the higher contact angles observed at elevated temperatures.

\section{Figure 4.}

\subsection{IR spectroscopy}

IR spectra are shown in Figure 4(b). PDEGMA exhibits distinctive peaks at 2928 and $2881 \mathrm{~cm}^{-1}$, which represent the $\mathrm{C}-\mathrm{H}$ stretching vibrations of $\mathrm{CH}_{3}$ and $\mathrm{CH}_{2}$, respectively. The strong peak at $1728 \mathrm{~cm}^{-1}$ results from the stretching vibration of $395 \mathrm{C}=\mathrm{O}$. Another characteristic peak at $1111 \mathrm{~cm}^{-1}$ corresponds to $\mathrm{C}-\mathrm{O}$ stretching. The spectrum of (PLL-CL) fibers (S4) shows features at 2948 and $1757 \mathrm{~cm}^{-1}$, arising from the stretching of $-\mathrm{CH}_{3}$ and $\mathrm{C}=\mathrm{O}$, respectively. Two peaks at 1183 and $1093 \mathrm{~cm}^{-1}$ are caused by the stretching of C-O-C. The IR spectrum for CIF shows distinct peaks at 
1589,1544 and $1500 \mathrm{~cm}^{-1}$ from stretching vibrations of the benzene ring. The peak at $4003046 \mathrm{~cm}^{-1}$ corresponds to $\mathrm{O}-\mathrm{H}$ stretching. $\mathrm{C}=\mathrm{O}$ stretching is visible at $1616 \mathrm{~cm}^{-1}$. Considering the spectra of S2 and S6, both show two adjacent peaks at 1757 and 1729 $\mathrm{cm}^{-1}$ from the stretching vibrations of $\mathrm{C}=\mathrm{O}$, which can be explained by the presence of PEDGMA and P(LLA-CL). The IR spectra of the other fibers similarly contain the major features from their polymer constituents. The drug-loaded S6 material has an 405 additional feature at $1619 \mathrm{~cm}^{-1}$. This corresponds to CIF, demonstrating that CIF was incorporated into the S6 nanofibers. Similarly, the other drug-loaded systems, S5, S7 and S8, also show additional peaks at $1619 \mathrm{~cm}^{-1}$, confirming the presence of CIF.

\subsection{In vitro release behavior}

410 The loadings of CIF in the fibers (S5, S6, S7 and S8) were determined prior to the investigation of their release behavior (Table 2). It was determined in all cases that more than $85 \%$ of the theoretical loading of CIF was present in the fibers, which demonstrates that most of the drug could be incorporated into the fibers successfully. It is thought that the remaining CIF may have crystallized out of solution onto the

415 needle and/or the walls of the syringe, owing to the very low solubility of this drug. The CIF release profiles from the fibers are depicted in Figure 4(c). The cumulative release percentage is expressed as a percentage of the measured loading. CIF is freed from the S8 ((P(LLA-CL) and CIF) fibers with an initial burst release of $40 \%$ during the first $10 \mathrm{~h}$, followed by further gradual release over more than $200 \mathrm{~h}$. After 420 immersion for $220 \mathrm{~h}$, the release of CIF reached a plateau at ca. $79 \%$. With regard to the composite nanofibers (S5, S6 and S7), all of them also exhibit a burst release of approximately $45 \%$ in the first $10 \mathrm{~h}$, after which gradual release occurs over about 170 hours. With increasing amounts of PDEGMA in the fibers, there appears to be a very slight increase in the CIF release rate. The cumulative CIF release percentages of

425 S5, S6 and S7 reach around $85 \%, 83 \%$ and $81 \%$, respectively, higher than the value for S8, the sample without PDEGMA.

The initial bursts of release observed in all the samples are the result of the presence of some CIF at the surface of the fibers. It has been shown conclusively in the 
literature that drug release from polymers in vitro is caused by diffusion, polymer erosion or a combination of both, and that release from a hydrophilic carrier tends to be faster than that from a hydrophobic material (Lin et al., 2012; Dong et al., 2008). The wettability of S5 is the highest of the four samples at $37{ }^{\circ} \mathrm{C}$, followed by S6, S7 and S8. This mirrors the order of release rates, where we see S5 $>$ S6 $>$ S7 $>$ S8. At the end of the release period, it can be seen that over $15 \%$ of the incorporated CIF was not freed into the release medium. It is believed that some CIF is encapsulated deep inside the fibers, especially with S8, which has the most hydrophobic properties. These results thus demonstrate that the PDEGMA/ P(LLA-CL) blend fibers can deliver sustained release of CIF over more than 7 days. Given this promising data, the fibers could extend the usage period and reduce the replacement frequency of wound 440 dressing materials.

Table 2. The loadings of CIF in the fibers, expressed as a percentage of the theoretical loading. Data are reported as mean \pm S.D. from three independent experiments.

\begin{tabular}{ccccc}
\hline Sample & S5 & S6 & S7 & S8 \\
& $(\mathbf{1 : 1 + C I F )}$ & $(\mathbf{1 : 2 + C I F )}$ & $(\mathbf{1 : 3 + C I F )}$ & $(\mathbf{P}(\mathbf{L L A}-\mathbf{C L})+\mathbf{C}$ \\
\hline CIF loading & $86.6 \pm 5.7$ & $87.3 \pm 5.1$ & $85.6 \pm 6.9$ & $86.9 \pm 5.5$ \\
$(\%)$ & & & & \\
\hline
\end{tabular}

\subsection{Cell growth and detachment}

It is reported that fibroblast cells are essential for wound healing, so L929 cells were chosen to test the cytocompatibility of the fibers (Cook et al., 2000). As a control, the

450 viability cell of a $0.9 \% \mathrm{CIF}$ solution (providing the same drug concentration as the fibers) was also explored. The results for this control experiment are shown in the Supporting Information (Figure S1). It can be seen that the number of cells increases 
continuously from 1 to 5 days, and at day 1 and 3 the untreated and CIF-treated results are indistinguishable. After 5 days, there are somewhat fewer cells present after CIF treatment. Overall, these data indicate that CIF has generally good cytocompatibility with this cell line.

As is depicted in Figure 5(a), the numbers of cells incubated on the fibers also increases from day 1 to day 5 under all conditions tested. The cell viabilities observed with all the fibers are similar, and they are significantly greater than those of the negative control (cover slips) especially on the $1^{\text {st }}$ and $5^{\text {th }}$ day $(\mathrm{p}<0.05)$. The amount of PDEGMA in the fibers has no major influence on their cytocompatibility.

The percentages of cells remaining attached to the cover slips after a treatment at $25^{\circ} \mathrm{C}$ are shown in Figure 5(b). It is clear that with blank coverslips and with coverslips coated in S8 (PLLA/CIF only), virtually $100 \%$ of the cells present remained attached after the $25{ }^{\circ} \mathrm{C}$ treatment. In contrast, with the PDEGMA-containing systems some of the cells can be detached by exposure to a temperature below the LCST. S5, which has the highest PDEGMA content, has the lowest percentage of cells remaining attached, at only $35.2 \%$. Significantly more cells remained attached to the S7 fibers $(76.44 \% ; \mathrm{p}<0.05)$. During the standard incubation at $37{ }^{\circ} \mathrm{C}$, above the LCST of PDEGMA, polymer-polymer interactions dominate, creating a hydrophobic surface on the blend fibers. P(LLA-CL) is also a hydrophobic polymer. Hence, adsorption of extracellular matrix (ECM) proteins onto the surface of the fiber mats is possible, which promotes cell adhesion (Brun-Graeppi et al., 2010; Cole et al., 2009). The treatment at $25{ }^{\circ} \mathrm{C}$ is below the LCST of 475 PDEGMA, and thus polymer-water interactions lead to a hydrophilic surface on the blend fibers. It is more difficult for proteins to adsorb onto such a hydrophilic surface (Chen et al., 2005; Okano et al., 1995), which causes the cells to become detached. The greater the amount of PDEGMA present in the fibers the more significant this effect becomes, and thus the larger the number of cells which are detached.

480 When the detached cells are transferred to a fresh 6-well plate and incubated at $37^{\circ} \mathrm{C}$, they can reattach themselves to the plate surface and grow normally (Figure 5(c)). This indicates that the detached cells have good viability, with no evidence of 
accelerated cell death observed after the detachment process. The composite PDEGMA/P(LLA-CL) fibers could thus be used as thermosensitive wound dressings: application of a reduced-temperature treatment inhibits the adhesion of cells and could be implemented to reduce secondary injuries.

\section{Figure 5}

\subsection{Antibacterial activity}

CIF is a fluoroquinolone, and is one of the most widely used antibiotics because of its low minimal inhibitory concentration for bacteria. The frequency of spontaneous resistance to CIF is also very low (Yu et al., 2006; Dillen et al., 2004). Control experiments were first performed to verify the antibacterial activity of CIF against $E$. coli and S. Aureus (see Figure S2): as is widely reported, this drug was extremely effective in preventing bacterial growth, with inhibition after 3 days calculated to be $87.5 \pm 6.2 \%$ and $84.6 \pm 2.8 \%$ against $E$. coli and $S$. Aureus respectively.

The results of antibacterial activity assays with the fibers are summarized in Table $\mathbf{3}$, with exemplar images given for S2 (with a PDEGMA to P(LLA-CL) ratio of 1:2) and

500 S6 (analogous to S2, but CIF-loaded) in Figure 6. A clear inhibition zone within and around the drug loaded S6 fibers can be seen after an overnight incubation of the agar plate at $37^{\circ} \mathrm{C}$, for both the Gram-positive and Gram-negative bacteria. However, these inhibition zones do not appear with the fibers without CIF (S2). The inhibition zones for E. coli and S. aureus after $24 \mathrm{~h}$ are $5.35 \pm 0.51 \mathrm{~cm}$ and $5.21 \pm 0.44 \mathrm{~cm}$, respectively. The complete date in Table 3 clearly demonstrate that the inhibition zones of all the fibers loaded with CIF (S5-S8) are similar after $24 \mathrm{~h}$, indicating comparable antibacterial effects, and inhibition zones cannot be seen in the groups without CIF (S1-S4).

After incubation for 72 hours, the inhibition zones are slightly larger than those

510 observed after $24 \mathrm{~h}$, indicating the antibacterial activity of the fibers persists for at least three days. This can be attributed to the drug release behavior of the fibers: as 
discussed above, CIF is released from the fibers gradually over more than 150 hours. It is clear that, given the lack of inhibitory zones in the CIF-free fibers, that the antibacterial activity of the drug-loaded systems is caused by CIF. The ability of

515 wound dressings to avoid infection by exogenous organisms is regarded as a requirement for an efficacious product, and hence the PDEGMA/P(LLA-CL) nanofibers loaded with CIF can potentially be applied as antibacterial wound dressing materials.

Table 3. Inhibition zone diameters for the fibers. Data are reported as mean \pm S.D. from 5 independent experiments.

\begin{tabular}{ccccc}
\hline Sample & \multicolumn{2}{c}{ Inhibition zones against } & \multicolumn{2}{c}{ Inhibition zones against $S}$. \\
& \multicolumn{2}{c}{$\boldsymbol{E . c o l i}(\mathbf{c m})$} & \multicolumn{2}{c}{ aureus $(\mathbf{c m})$} \\
\hline & 24 hours & 72 hours & 24 hours & 72 hours \\
S1 & --- & --- & --- & --- \\
S2 & --- & --- & --- & -- \\
S3 & --- & --- & --- & -- \\
S4 & ---- & --- & - \\
S5 & $5.52 \pm 0.63$ & $5.64 \pm 0.43$ & $5.24 \pm 0.38$ & $5.32 \pm 0.37$ \\
S6 & $5.35 \pm 0.51$ & $5.45 \pm 0.81$ & $5.21 \pm 0.44$ & $5.62 \pm 0.53$ \\
S7 & $5.39 \pm 0.58$ & $5.57 \pm 0.25$ & $5.45 \pm 0.82$ & $5.55 \pm 0.18$ \\
S8 & $5.28 \pm 0.12$ & $5.40 \pm 0.26$ & $5.52 \pm 0.66$ & $5.61 \pm 0.83$ \\
\hline
\end{tabular}

\section{Figure 6}

3.9 Fiber degradation and water uptake in vitro

The degradation of the fibers was explored in vitro, in order to validate that they would persist in physiological media for long enough to effect wound healing. The results are given in Figure S3. The weight loss after 6 weeks is found to be less than 
$53010 \%$. Further, the $\mathrm{pH}$ of the buffer used for degradation remained largely constant over this period, declining only from ca. 7.25 to 6.75 . This indicates that only small amounts of lactic acid are being feed from the fibers, and thus that local inflammation from this effect is likely to be minimal. The fiber mats are found to be able to uptake large amounts of water (between 80 and $170 \%$ by weight; Table S1). This is

535 somewhat less than the gauze (approx.. $180 \%$ ), but nevertheless demonstrates the porosity of the fibers and their abilities to absorb wound extrudates.

\subsection{Macroscopic observation of the wound healing process}

The ability of the fibers to aid wound healing in vivo was explored with S6 and S8, with a commercial gauze (Shanghai Yinjing Meidical Supplies Company Ltd), the same gauze immersed in a $0.9 \% \mathrm{w} / \mathrm{v}$ CIF solution and a $0.9 \% \mathrm{w} / \mathrm{v}$ CIF solution used as control groups. Figure 7 shows representative images of the wound healing process. The rats awoke around 3 hours after the operation, and appeared healthy. In the groups treated with gauze, gauze soaked with CIF and CIF solution (Figure 7(a),

545 (b) and (c)), thicker scabs or bleeding can be seen, resulting from local inflammation. These groups additionally demonstrate slower wound healing than those treated with S6 and S8.

Among the 5 groups, the S6 dressings were easiest to remove from the wounds after treatment with cold saline. Moreover, S6 shows the best healing performance. After 5 550 days, the wound treated with S6 was completely covered by a scab and there were no signs of infection. After 14 days, the area of the wound was much reduced. At the end of the period (21 days), the wound was largely scab-free and mostly filled with regenerated skin and hairs. This process is clearly occurring more rapidly with S6, with S8 somewhat less effective and the wounds covered with gauze only healing 555 noticeably more slowly.

The relative wound areas of the various groups after operation are given in Figure 8. The results are in accord with those in Figure 7. After 5 days, the wound areas are almost the same as immediately post-operation with all groups. Subsequently the wound areas decline rapidly. After 21 days, $\mathrm{S} 6$ shows the lowest area, at $5.7 \pm 0.8 \%$ 
560 of the original area, with S8 $(14.3 \pm 1.6 \%)$ performing significantly less well $(\mathrm{p}<$ 0.05). Because of the presence of PDEGMA in S6, these fibers could be removed from the wounds more easily than S8. As a result, the induction of secondary injuries during the changing of dressings is reduced and the wound healing is faster.

The wound areas of the groups treated with gauze, gauze soaked with CIF solution, 565 and CIF solution remain high even after 21 days, at $30.8 \pm 2.2 \%, 26.5 \pm 1.9 \%$ and $28.3 \pm 1.7 \%$, respectively. The presence of CIF in the gauze clearly helped to speed up the wound healing process, and the application of the gauze covered and protected the wounds. However, healing in these groups is significantly slower than in those treated with S6 and S8 (p < 0.05).

570 The degradation of the fiber mats was also studied in vivo (see Figures S4 and S5). The S6 and S6 systems undergo less than $10 \%$ mass loss over 4 weeks of use as a wound dressing (Figure S4). SEM images (Figure S5) show that there are no major changes in morphology after the fibers are placed on and removed from skin, be this healthy skin or a wound. Only a small amount of swelling can be observed.

575 It has been reported that there are a number of advantages in using electrospun nanofibers as wound dressings, resulting from their high surface area-to-volume ratio, hemostasis, semi-permeability, functional ability, etc (Zahedi et al., 2010; Zou et al., 2011). In particular, the thermoresponsive behavior of S6 is thought to facilitate cell detachment, reducing secondary injuries and hence leading to improved healing 580 performance over S8. The water uptake of S6 is somewhat greater than that of S8 (see Table S1), which may also contribute to its improved performance. However, both sets of fibers absorb less water than the commercial gauze, and the latter performs worse in healing. Thus, water uptake is not thought to be the key factor in determining the efficacy of healing. Moreover the sustained release of CIF from the

585 fibers prevents bacterial contamination and thus also contributes to the faster healing of wounds. Therefore, we can be confident that the PDEGMA/P(LLA-CL) nanofibers loaded with CIF are promising materials for wound healing in rats in vivo. 


\section{Figure 7}

\section{Figure 8}

3.11 Toxicological evaluation, histological examinations and protein expression None of the nanofiber dressings applied showed any hypersensitivity reactions on the skin. There was no sign of erythema, eschar or edema, which indicates the safety of the fiber as wound dressings. Toxicological results obtained on blood samples are detailed in Table S2. In all cases, the values obtained for animals treated with the fiber wound dressings are almost identical to an untreated control. These findings make it clear that the fibers are safe to use as wound dressings.

600 Histological data are presented in Figure 9. New epidermis, marked by rectangles, could be observed at the edge of the wound in all groups. In the gauze group (Figure 9(a)), the epidermis is very thin and irregular, and inflammation (black arrows) can be seen in the sections. The groups treated with gauze soaked in $0.9 \%$ CIF solution and CIF solution (Figure 9(b) and (c)), have wounds which are completely epithelized,

605 but the epidermis is not continuous and is irregular in its thickness. In contrast, the regeneration of the epidermis in the fiber groups (Figure 9(d) and (e)) is much more complete. It can be seen that the basal cells (white arrows) are uniform in shape and are arranged close together. The continuity and regularity of the epidermis are closer to the normal skin (Figure 9(f)) than any of the other treatment groups. Compared to

$610 \mathrm{~S} 8$, the thickness of the epidermis in S6 is more uniform. Further, the horny layers (marked by a circle in Figure 9) in the S6 group are dense and more interconnected. Again, these are closed to the normal skin than is seen with the other groups, although it should be noted that some fragments were induced while preparing the histological sections. The horny layers in S8 are not at all obvious, which may result from the

615 secondary injuries arising while the dressings were replaced. These results agree fully with the outcomes of the macroscopic observations discussed above, demonstrating the enhanced healing performance of S6 over the other groups. 


\section{Figure 9}

620

To probe the mechanism underlying the acceleration of wound healing by the fibers, the expression of CD34 and collagen type I in the regenerated tissues of all the in vivo groups were evaluated by Western blotting assays, using $\beta$-actin as protein loading control. After 5 days the expression of CD34, a marker of vascular endothelial cells, is

625 low in the groups treated with gauze (Figure 10(a)), gauze soaked in CIF (Figure 10(b)) and CIF solution (Figure 10(c)). In contrast, at this time point CD34 is highly expressed in rats treated with S8 (Figure 10(d)) and S6 (Figure 10(e)). After 14 days, CD34 is highly expressed in the groups treated with gauze with and without CIF or with the CIF solution, while the amount present in the fiber-treated wounds has

630 declined. 21 days post-operation, the expression of CD34 had declined in all treatment groups. CD34 expression in normal skin remains low during the entire period, as it is not expressed in skin without wounds.

The expression of CD34 can be related both to the release of CIF and the other wound-healing attributes of the fibers. The release of CIF from the nanofibers is

635 sustained, which can prevent the infection of wounds in the early stage of healing. As a result, a large number of vascular endothelial cells proliferate and migrate into the wound region to participate in the regeneration of blood capillaries and promote healing (Wilgus, 2008). The ability of the fibers to absorb extrudates and (in the case of S6) encourage cell detachment is synergistic with this and results in early wound

640 healing and upregulation of CD34. In contrast, while the CIF can still exert its antibacterial properties in the control groups, these lack the other advantageous properties of the fibers, and hence CD34 levels peak later.

Collagen is the main constituent of the extracellular matrix secreted by fibroblasts, and collagen type $\mathrm{I}$ is a major component of regenerated skin (Yang et al., 2011).

645 Hence, high levels of collagen type I expression can be seen in the normal skin at each time point (see Figure 10). During the wound healing process, collagen type I 
expression continuously increased in all the experimental groups. Rats treated with S6 (Figure 10(e)) exhibit the highest amount of at each time point, followed by S8. By the $21^{\text {st }}$ day, the collagen band is as intense from the S6 group as in normal skin, showing that healing is almost complete. These results support the macroscopic wound appearance study and the histological staining data. Therefore, all the results of the in vivo experiments are in agreement and demonstrate that the S6 fibers exhibited better healing performance than all the other groups.

655

\section{Figure 10}

\section{Conclusions}

660 In this study, the thermosensitive polymer PDEGMA (poly(di(ethylene glycol) methyl ether methacrylate) was first synthesized and characterized. Composite nanofibers of PDEGMA and P(LLA-CL) (poly(l-lactic acid-co- $\varepsilon$-caprolactone)) were successfully prepared by electrospinning, and analogous ciprofloxacin (CIF)-loaded fibers were also generated. Electron microscopy showed that smooth fibers were produced,

665 although a small number of beads can be seen in the ciprofloxacin-loaded fibers. The fiber diameters decrease with increases in the proportions of PDEGMA and CIF in the formulations. X-ray diffraction showed that CIF was present in the amorphous physical form after electrospinning. The water contact angle of the composite nanofibers increases abruptly when the temperature is increased through the lower

670 critical solution temperature of $32{ }^{\circ} \mathrm{C}$. The fibers thus undergo a rapid hydrophilic/hydrophobic transition at this point. Sustained release of CIF from the drug-loaded fibers was observed to proceed over more than 160 hours, and the fibers were able to promote the adhesion and proliferation of L929 fibroblasts. By reducing the culture temperature, cells could be detached from the PDEGMA-containing fibers. 
675 The composite fibers loaded with CIF also inhibited the growth of E. coli and $S$. aureus. In vivo tests demonstrated that PDEGMA/PLLA fibers loaded with CIF had better wound healing performance compared with commercial gauze or PLLA/CIF fibers. Our findings therefore show that PDEGMA / P(LLA-CL) / ciprofloxacin fibers can act as potent wound dressing materials.

680

\section{Acknow ledgements}

This investigation was supported by the Biomedical Textile Materials "111 Project" from the Ministry of Education of China (No. B07024), and the UK-China Joint Laboratory for Therapeutic Textiles (based at Donghua University).

685

\section{References}

Akiyama, Y., Kikuchi, A., Yamato, M., Okano, T., 2004. Ultrathin poly(N-isopropylacrylamide) grafted layer on polystyrene surfaces for cell adhesion/detachment control. Langmuir, 20, 5506-5511.

690 Becer, C. R., Hahn, S., Fijten, M. W., Thijs, H. M., Hoogenboom, R., Schubert, U. S., 2008. Libraries of methacrylic acid and oligo (ethylene glycol) methacrylate copolymers with LCST behavior. J. Polym. Sci. A Polym. Chem. 46, 7138-7147.

Behl, M., Razzaq, M. Y., Lendlein, A., 2010. Multifunctional Shape-Memory Polymers. Adv. Mater. 22, 3388-3410.

695 Boateng, J. S., Matthews, K. H., Stevens, H. N., Eccleston, G. M., 2008. Wound healing dressings and drug delivery systems: a review. J. Pharm. Sci. 97, 2892-2923.

Brun-Graeppi, A. K. A. S., Richard, C., Bessodes, M., Scherman, D., Merten, O. W., 2010. Thermoresponsive surfaces for cell culture and enzyme-free cell detachment. Prog. Polym. Sci. 35, 1311-1324.

Charernsriwilaiwat, N., Opanasopit, P., Rojanarata, T., Ngawhirunpat, T., 2012. Lysozyme-loaded, electrospun chitosan-based nanofiber mats for wound healing. Int. J. Pharm. 427, 379-384. 
Chen, S., Zheng, J., Li, L., Jiang, S., 2005. Strong resistance of phosphorylcholine 705 self-assembled monolayers to protein adsorption: insights into nonfouling properties of zwitterionic materials. J. Am. Chem. Soc. 127, 14473-14478.

Cho, Y. S., Lee, J. W., Lee, J. S., Lee, J. H., Yoon, T. R., Kuroyanagi, Y., Park, M. H., Kim, H. J., 2002. Hyaluronic acid and silver sulfadiazine-impregnated polyurethane foams for wound dressing application. J. Mater. Sci. Mater. Med. $710 \quad 13,861-865$.

Cole, M. A., Voelcker, N. H., Thissen, H., Griesser, H. J., 2009. Stimuli-responsive interfaces and systems for the control of protein-surface and cell-surface interactions. Biomaterials, 30, 1827-1850.

Cook, H., Stephens, P., Davies, K. J., Harding, K. G., Thomas, D. W., 2000. Defective 715 extracellular matrix reorganization by chronic wound fibroblasts is associated with alterations in TIMP-1, TIMP-2, and MMP-2 activity. J. Invest. Dermatol. $115,225-233$.

Dillen, K., Vandervoort, J., Van den Mooter, G., Verheyden, L., Ludwig, A., 2004. Factorial design, physicochemical characterisation and activity of ciprofloxacin-PLGA nanoparticles. Int. J. Pharm. 275, 171-187.

Dimitrov, I., Trzebicka, B., Müller, A. H., Dworak, A., Tsvetanov, C. B., 2007. Thermosensitive water-soluble copolymers with doubly responsive reversibly interacting entities. Prog. Polym. Sci. 32, 1275-1343.

Dong, Y., Zhang, Z., Feng, S. S., 2008. d- $\alpha$-Tocopheryl polyethylene glycol 1000 succinate (TPGS) modified poly (l-lactide)(PLLA) films for localized delivery of paclitaxel. Int. J. Pharm. 350, 166-171.

Gil, E. S., Hudson, S. M., 2007. Effect of silk fibroin interpenetrating networks on swelling/deswelling kinetics and rheological properties of poly (N-isopropylacrylamide) hydrogels. Biomacromolecules, 8, 258-264.

730 Han, S., Hagiwara, M., Ishizone, T., 2003. Synthesis of thermally sensitive water-soluble polymethacrylates by living anionic polymerizations of oligo (ethylene glycol) methyl ether methacrylates. Macromolecules, 36, 8312-8319. 
Heilmann, S., Küchler, S., Wischke, C., Lendlein, A., Stein, C., Schäfer-Korting, M., 2008. A thermosensitive morphine-containing hydrogel for the treatment of large-scale skin wounds. Int. J. Pharm. 444, 96-102.

Ishizone, T., Seki, A., Hagiwara, M., Han, S., Yokoyama, H., Oyane, A., Deffieux, A., Carlotti, S., 2008. Anionic polymerizations of oligo (ethylene glycol) alkyl ether methacrylates: effect of side chain length and $\omega$-alkyl group of side chain on cloud point in water. Macromolecules, 41, 2963-2967.

740 Li, H., Wang, M., Williams, G. R., Wu, J., Sun, X., Lv, Y., Zhu, L. M., 2016. Electrospun gelatin nanofibers loaded with vitamins A and E as antibacterial wound dressing materials. RSC Adv. 6, 50267-50277.

Lin, X., Tang, D., Cui, W., Cheng, Y., 2012. Controllable drug release of electrospun thermoresponsive poly(N-isopropylacrylamide)/

745 poly(2-acrylamido-2-methylpropanesulfonic acid) nanofibers. J. Biomed. Mater. Res. A, 100, 1839-1845

Li, X., Su, Y., Zhou, X., Mo, X., 2009. Distribution of Sorbitan Monooleate in poly (1-lactide-co- $\varepsilon$-caprolactone) nanofibers from emulsion electrospinning. Colloids Surf. B Biointerfaces, 69, 221-224.

750 Lutz, J. F., Weichenhan, K., Akdemir, Ö., Hoth, A, 2007. About the phase transitions in aqueous solutions of thermoresponsive copolymers and hydrogels based on 2-(2-methoxyethoxy) ethyl methacrylate and oligo (ethylene glycol) methacrylate. Macromolecules, 40, 2503-2508.

Lutz, J. F., 2008. Polymerization of oligo (ethylene glycol)(meth) acrylates: toward 755 new generations of smart biocompatible materials. J. Polym. Sci. A Polym. Chem. 46, 3459-3470.

Lutz, J. F., 2011. Thermo-Switchable Materials Prepared Using the OEGMA-Platform. Adv. Mater. 23, 2237-2243.

Maeda, Y., Kubota, T., Yamauchi, H., Nakaji, T., Kitano, H., 2007. Hydration changes of poly (2-(2-methoxyethoxy) ethyl methacrylate) during thermosensitive phase separation in water. Langmuir, 23, 11259-11265.

Newman, G. R., Walker, M., Hobot, J. A., Bowler, P. G., 2006. Visualisation of 
bacterial sequestration and bactericidal activity within hydrating Hydrofiber ${ }^{\circledR}$ wound dressings. Biomaterials, 27, 1129-1139.

765 Okano, T., Yamada, N., Okuhara, M., Sakai, H., Sakurai, Y., 1995. Mechanism of cell detachment from temperature-modulated, hydrophilic-hydrophobic polymer surfaces. Biomaterials, 16, 297-303.

Pelipenko, J., Kristl, J., Janković, B., Baumgartner, S., Kocbek, P., 2013. The impact of relative humidity during electrospinning on the morphology and mechanical properties of nanofibers. Int. J. Pharm. 456, 125-134.

Pham, Q. P., Sharma, U., Mikos, A. G., 2006. Electrospinning of polymeric nanofibers for tissue engineering applications: a review. Tissue Eng. 12, 1197-1211.

Rath, G., Hussain, T., Chauhan, G., Garg, T., Goyal, A. K., 2016. Development and characterization of cefazolin loaded zinc oxide nanoparticles composite gelatin 775 nanofiber mats for postoperative surgical wounds. Materials Science and Engineering: C, 58, 242-253.

Reddy, T. T., Kano, A., Maruyama, A., Hadano, M., Takahara, A., 2008. Thermosensitive transparent semi-interpenetrating polymer networks for wound dressing and cell adhesion control. Biomacromolecules, 9, 1313-1321.

780 Schild, H. G., 1992. Poly (N-isopropylacrylamide): experiment, theory and application. Prog. Polym. Sci. 17, 163-249.

Sun, K., Bligh, S. A., Nie, H. L., Quan, J., Zhu, L. M., 2014. Lectin recognizing thermoresponsive double hydrophilic glycopolymer micelles by RAFT polymerization. RSC Adv. 4, 34912-34921.

785 Su, Y., Li, X., Liu, S., Mo, X., Ramakrishna, S., 2009. Controlled release of dual drugs from emulsion electrospun nanofibrous mats, Colloids Surf. B Biointerfaces , 73, 376-381.

Tan, S. P., McLoughlin, P., O'Sullivan, L., Prieto, M. L., Gardiner, G. E., Lawlor, P. G., Hughes, H., 2013. Development of a novel antimicrobial seaweed extract-based hydrogel wound dressing. Int. J. Pharm. 456, 10-20.

Thakur, R. A., Florek, C. A., Kohn, J., Michniak, B. B., 2008. Electrospun nanofibrous polymeric scaffold with targeted drug release profiles for potential 
application as wound dressing. Int. J. Pharm. 364, 87-93.

Thomas, S., Loveless, P., Hay, N. P., 1988. Comparative review of the properties of six semipermeable film dressings. Pharm. J. 240, 785-787.

Thu, H. E., Zulfakar, M. H., Ng, S. F., 2012. Alginate based bilayer hydrocolloid films as potential slow-release modern wound dressing. Int. J. Pharm. 434, 375-383.

Unnithan, A. R., Barakat, N. A., Pichiah, P. T., Gnanasekaran, G., Nirmala, R., Cha, Y. S., Jung, C., El-Newehy, M., Kim, H. Y., 2012. Wound-dressing materials with antibacterial activity from electrospun polyurethane-dextran nanofiber mats containing ciprofloxacin HCl. Carbohydr. Polym. 90, 1786-1793.

Wang, L. S., Chow, P. Y., Phan, T. T., Lim, I. J., Yang, Y. Y., 2006. Fabrication and characterization of nanostructured and thermosensitive polymer membranes for wound healing and cell grafting. Adv. Funct. Mater. 16, 1171-1178.

805 Wilgus, T. A., 2008. Immune cells in the healing skin wound: influential players at each stage of repair. Pharmacol. Res. 58, 112-116.

Yang, J. M., Yang, S. J., Lin, H. T., Wu, T. H., Chen, H. J., 2008. Chitosan containing PU/Poly (NIPAAm) thermosensitive membrane for wound dressing. Mater. Sci. Eng. C, 28, 150-156.

810 Yang, Y., Xia, T., Zhi, W., Wei, L., Weng, J., Zhang, C., Li, X., 2011. Promotion of skin regeneration in diabetic rats by electrospun core-sheath fibers loaded with basic fibroblast growth factor. Biomaterials, 32, 4243-4254.

Yin, A., Zhang, K., McClure, M. J., Huang, C., Wu, J., Fang, J., Mo, X., Bowlin, G. L., Al-Deyab, S. S., El-Newehy, M., 2013. Electrospinning collagen/chitosan/poly 815 (L-lactic acid-co- $\epsilon$-caprolactone) to form a vascular graft: Mechanical and biological characterization. J. Biomed. Mater. Res. A, 101, 1292-1301.

Yu, H., Xu, X., Chen, X., Hao, J., Jing, X., 2006. Medicated wound dressings based on poly (vinyl alcohol)/poly (N-vinyl pyrrolidone)/chitosan hydrogels. J. Appl. Polym. Sci. 101, 2453-2463.

820 Zahedi, P., Rezaeian, I., Ranaei-Siadat, S. O., Jafari, S. H., Supaphol, P., 2010. A review on wound dressings with an emphasis on electrospun nanofibrous polymeric bandages. Polym. Adv. Technol. 21, 77-95. 
Zhang, Y., Lim, C. T., Ramakrishna, S., Huang, Z. M., 2005. Recent development of polymer nanofibers for biomedical and biotechnological applications. J. Mater. Sci. Mater. Med. 16, 933-946.

Zou, B., Li, X., Zhuang, H., Cui, W., Zou, J., Chen, J., 2011. Degradation behaviors of electrospun fibrous composites of hydroxyapatite and chemically modified poly (DL-lactide). Polym. Degrad. Stab. 96, 114-122. 
Figures

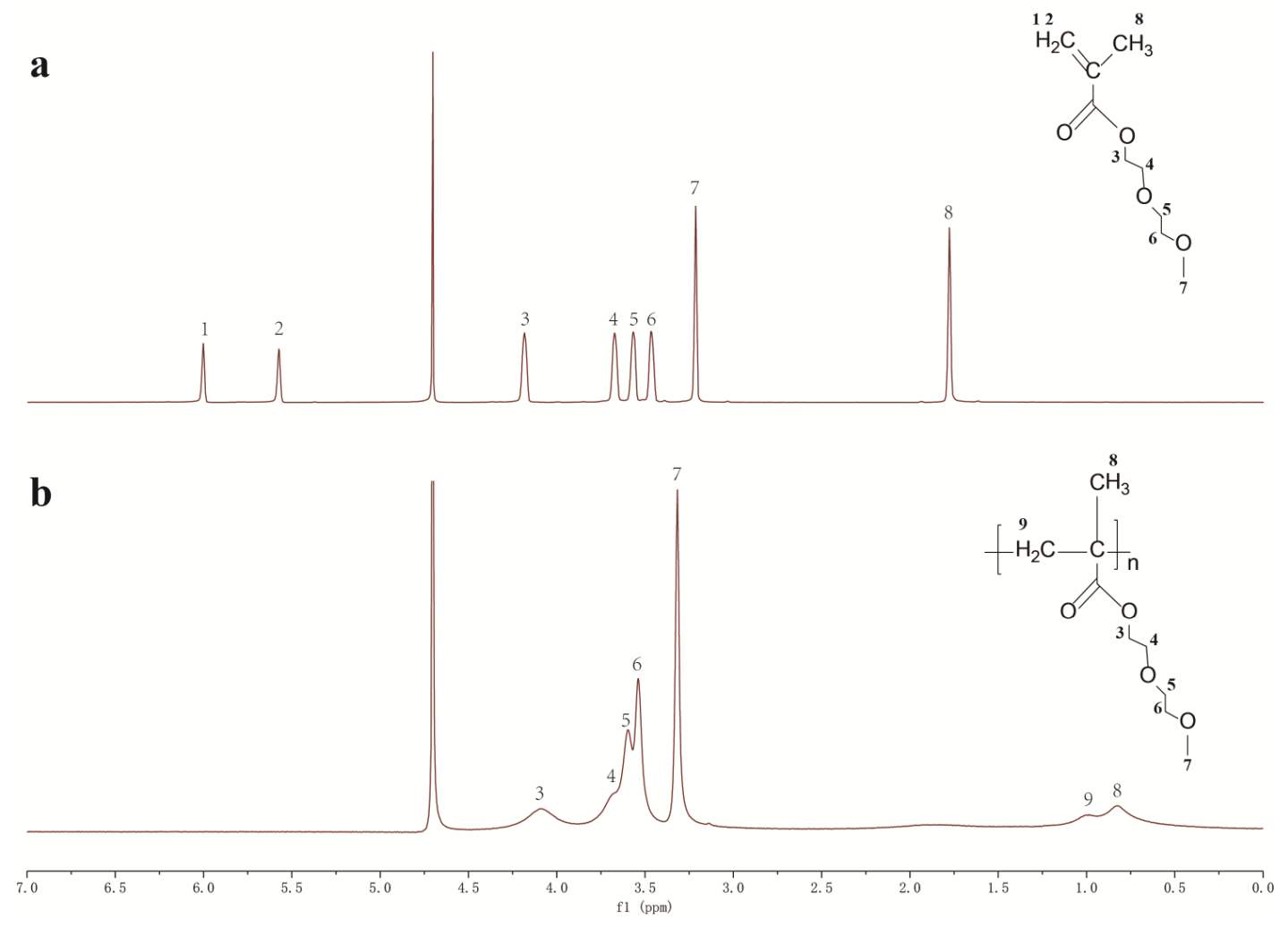

Figure 1. ${ }^{1} \mathrm{H}$ NMR spectra of (a) DEGMA and (b) PDEGMA 835 


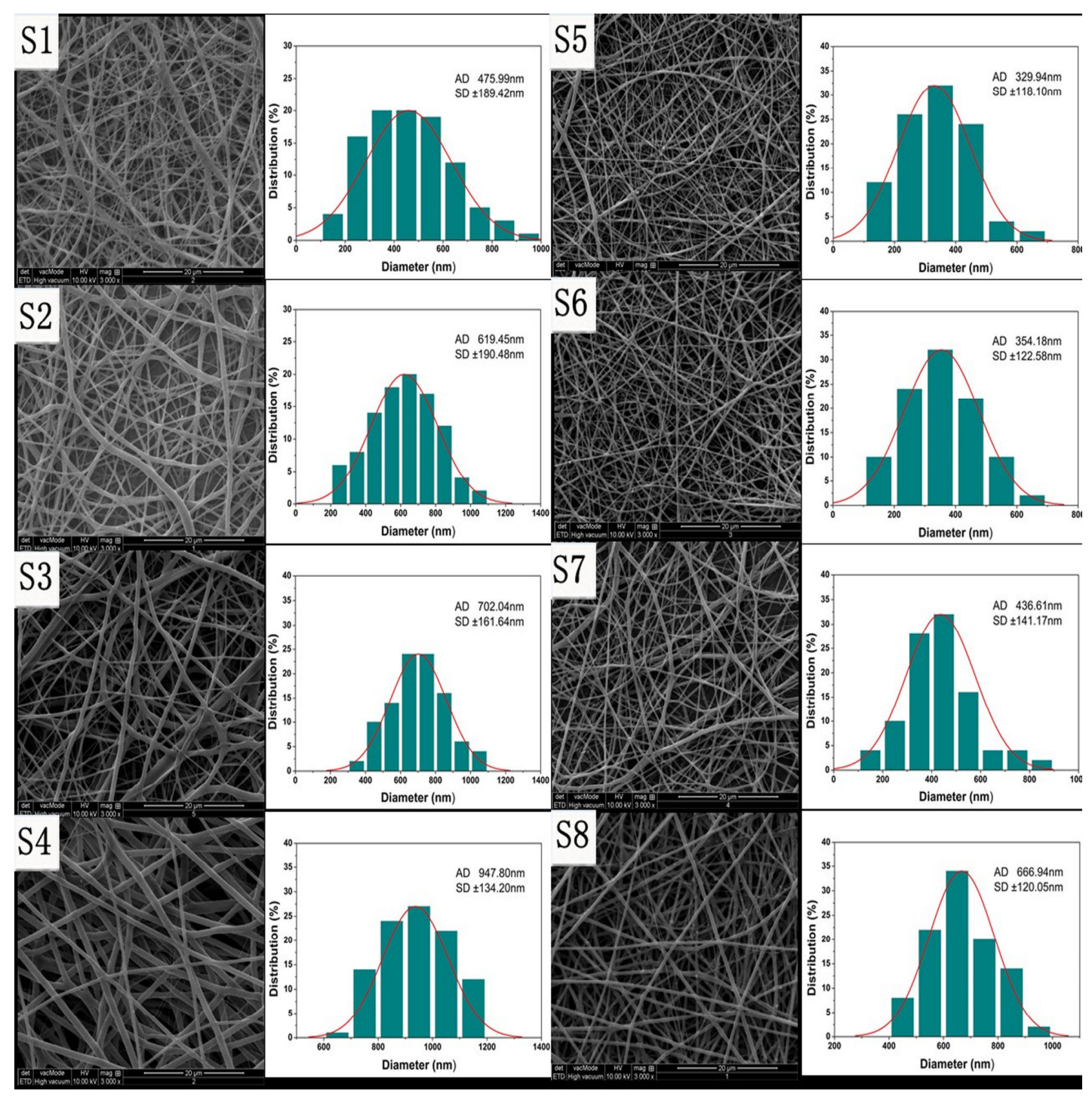

Figure 2. SEM images and diameter distributions of the fibers prepared in this work. 


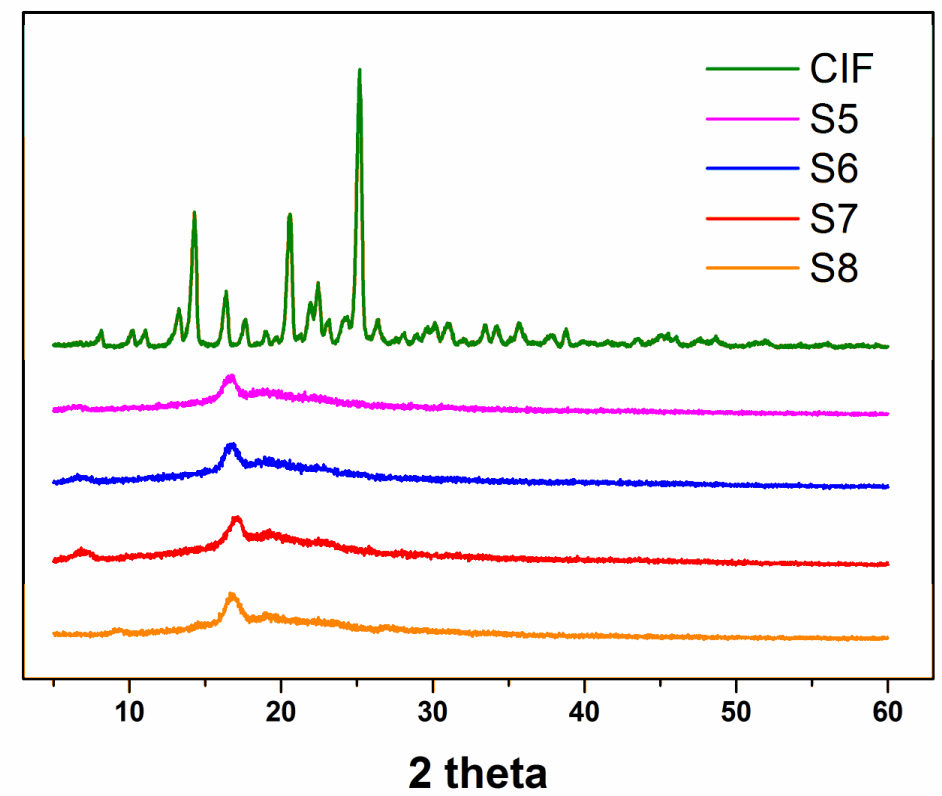

Figure 3. XRD patterns of CIF and the CIF loaded nanofibers 840 


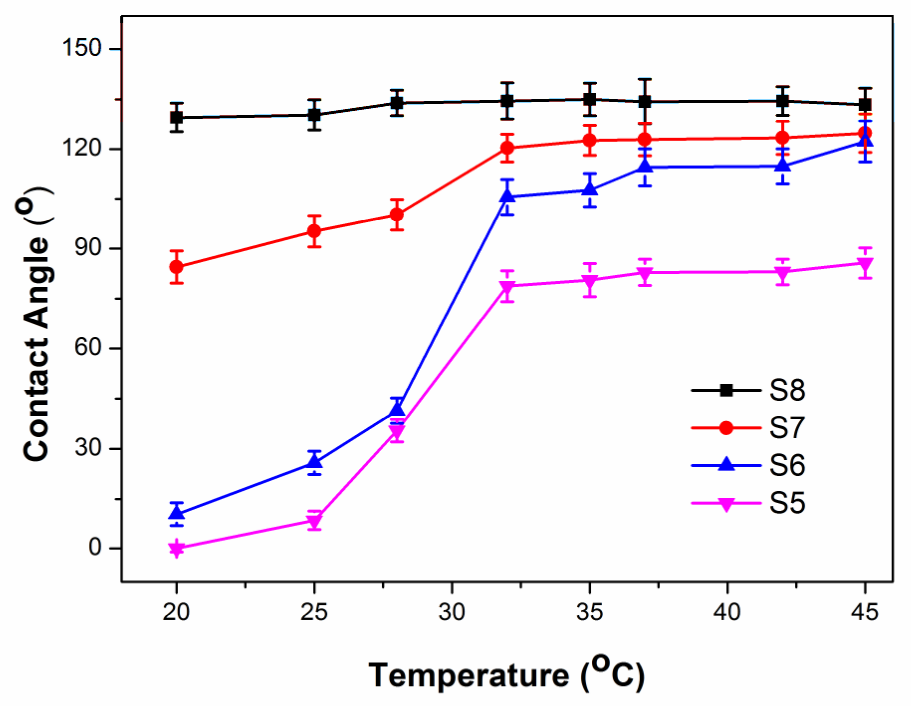

(a)

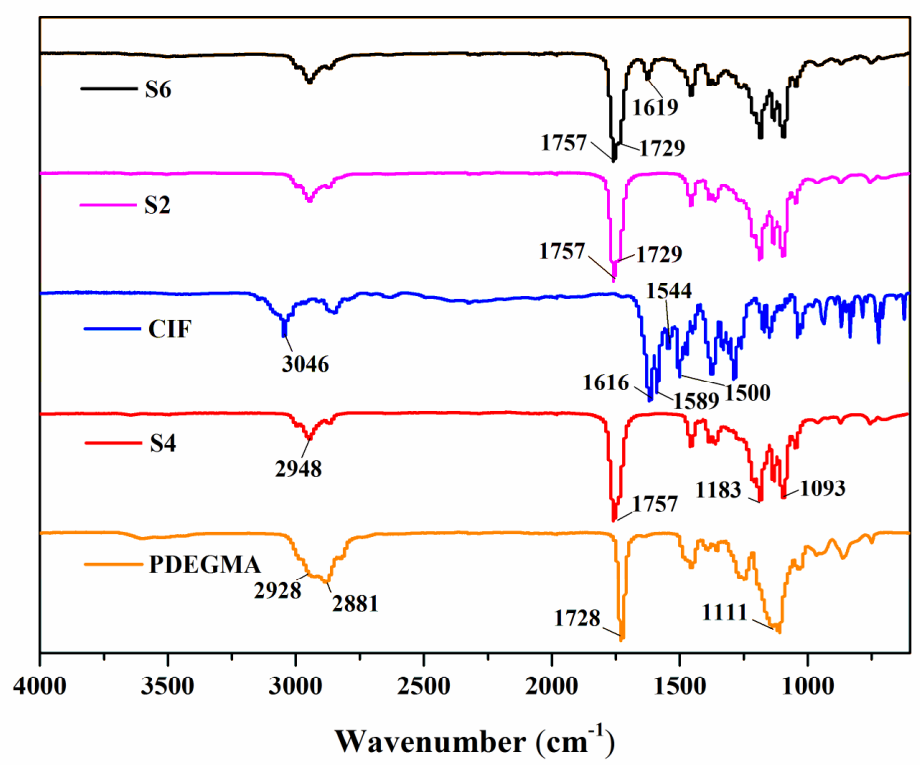

(b) 


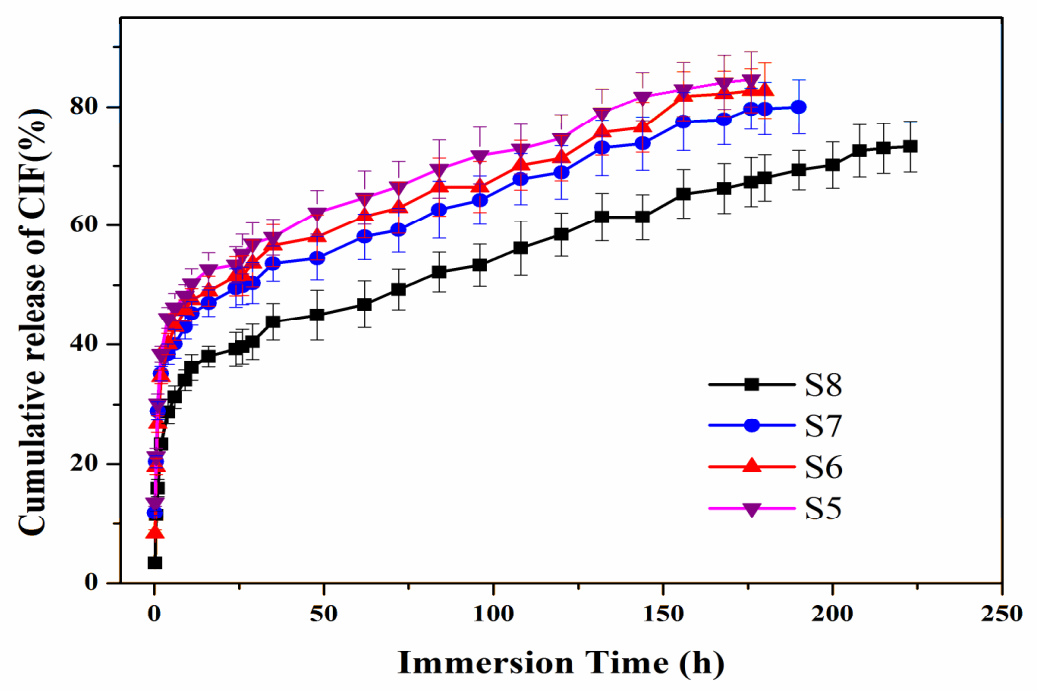

845

(c)

Figure 4. a) The temperature dependence of the fibers' water contact angles; b) FTIR spectra of PDEGMA, CIF and selected fibers (S2, S4 and S6); and, c) The in vitro release profiles of CIF from the drug-loaded fibers. Data are reported as mean \pm S.D.

850 from three independent experiments. 

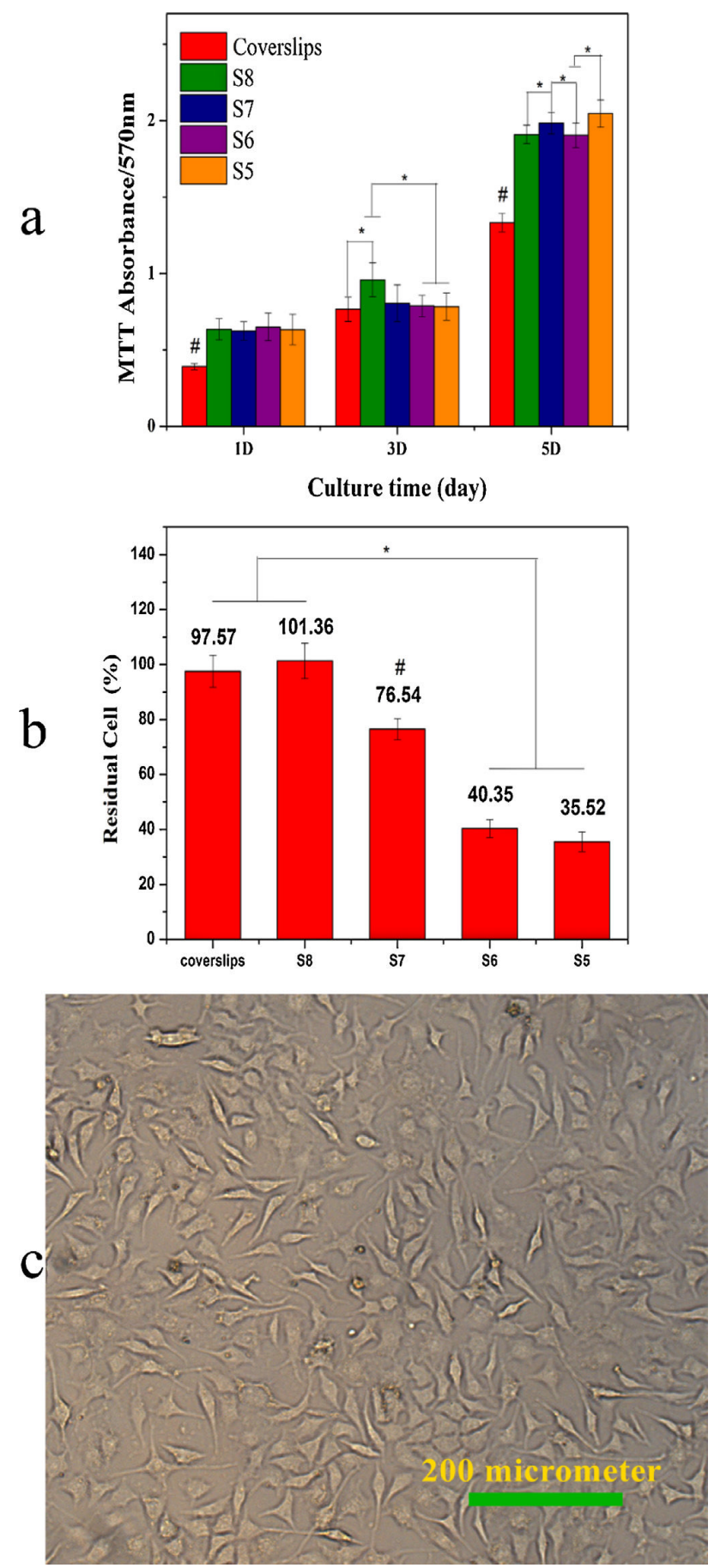

Figure 5. a) Cell viability after exposure to the electrospun fibers for 1, 3, or 5 days; b) The percentage of L929 fibroblasts remaining attached to the fibers after treatment at $85525^{\circ} \mathrm{C}$; and, c) A micrograph of L929 fibroblasts detached from the S6 fibers and cultured in a fresh plate at $37{ }^{\circ} \mathrm{C}$ (magnification: $\times 100$ ). Data in Figure 3 (a) and (b) are from three independent experiments, and are reported as mean \pm S.D. * indicates a significant difference between two groups $(\mathrm{p}<0.05)$ and \# indicates a significant difference from all other groups $(\mathrm{p}<0.05)$. 


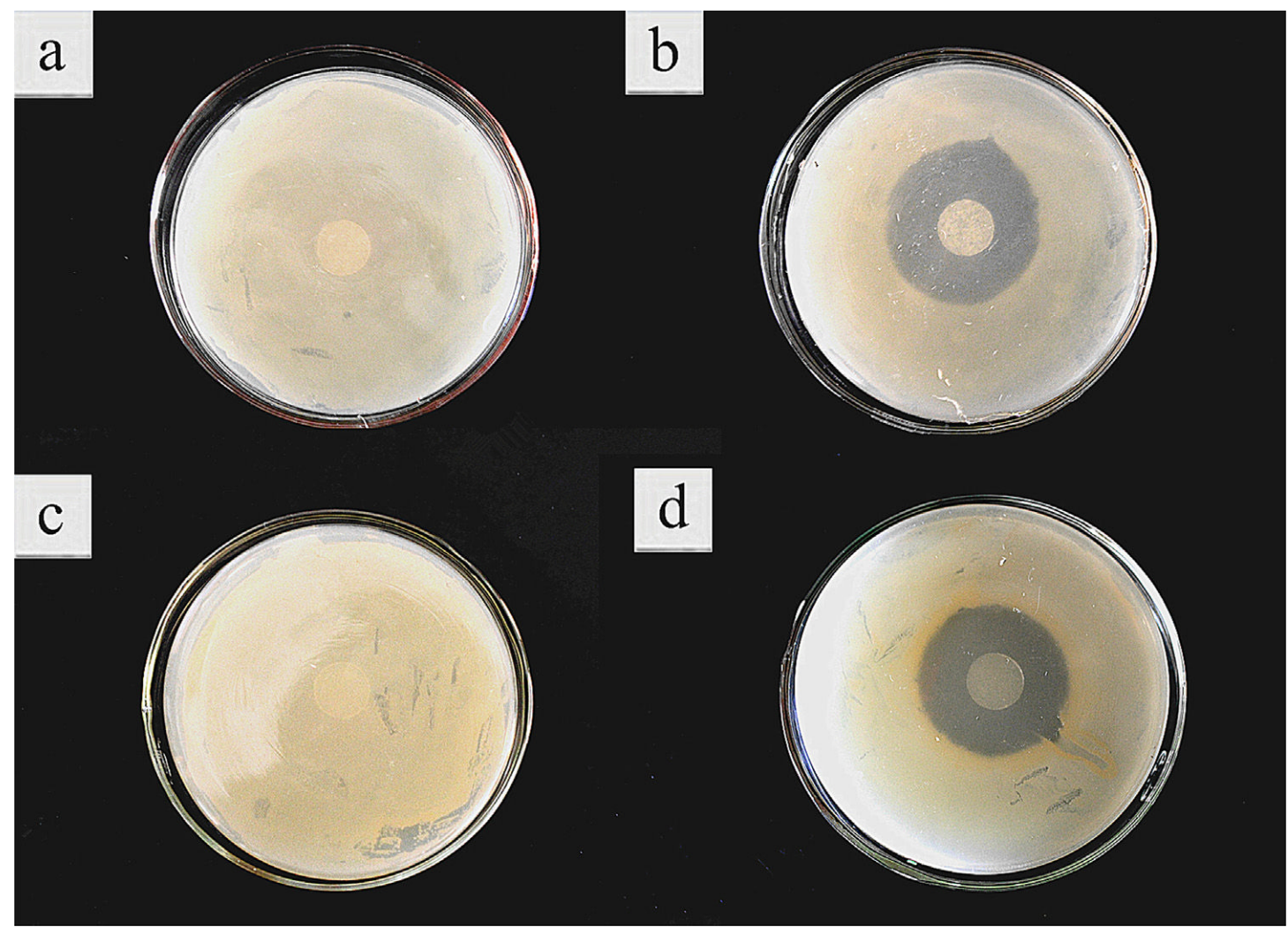

Figure 6. Bactericidal activity of selected fiber samples. Representative photographs of a) S2 against E. coli; b) S6 against E.coli; c) S2 against S. aureus; and, d) S6 against $S$. aureus, taken from 5 replicate experiments. 

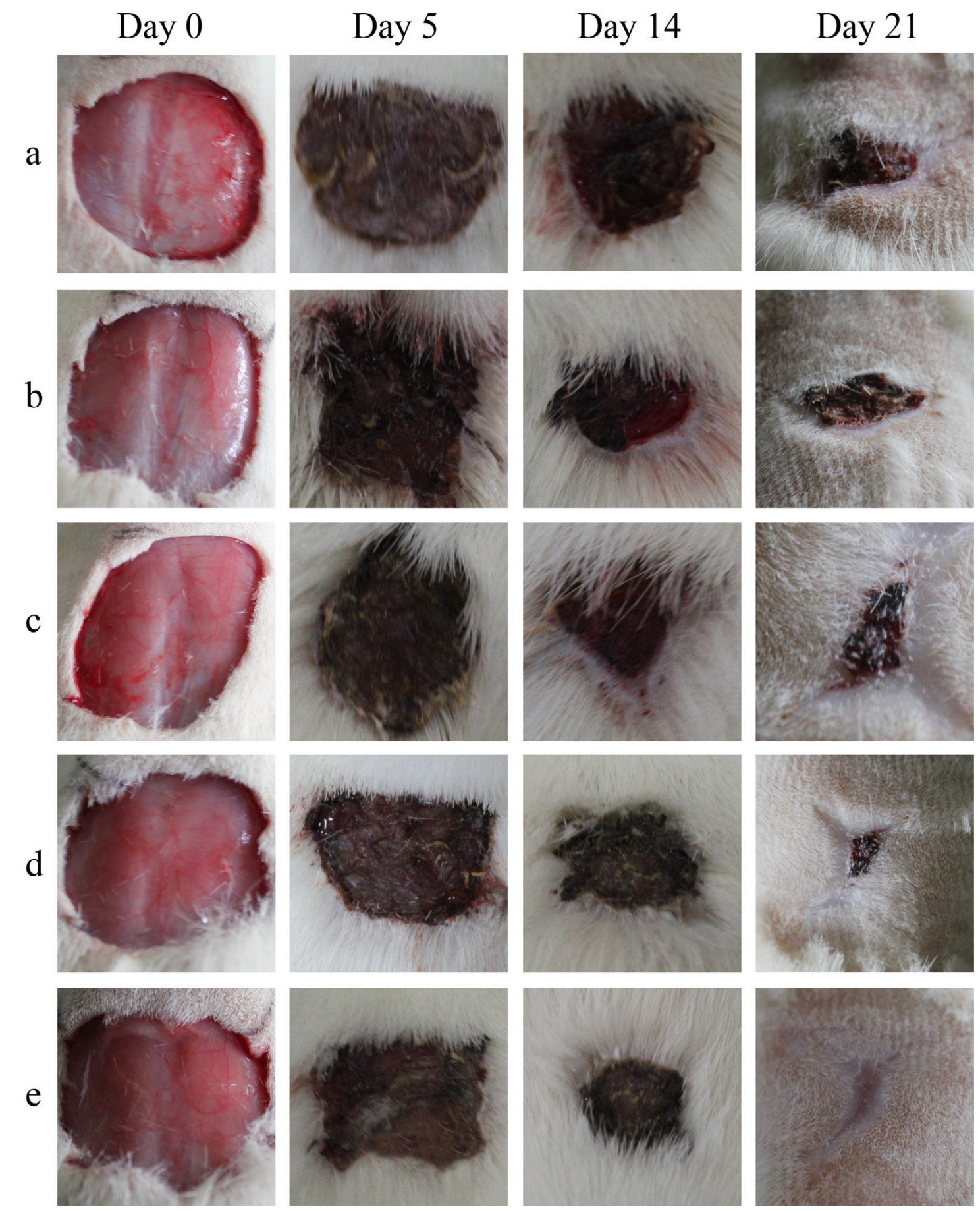

Figure 7. Representative images of skin wounds after treatment with selected fibers for $0,5,14$ and 21 days post-operation. The wounds were treated with (a) a commercial gauze alone; (b) the same type of gauze soaked with a $0.9 \%$ CIF solution; (c) CIF solution; (d) S8; and, (e) S6. 


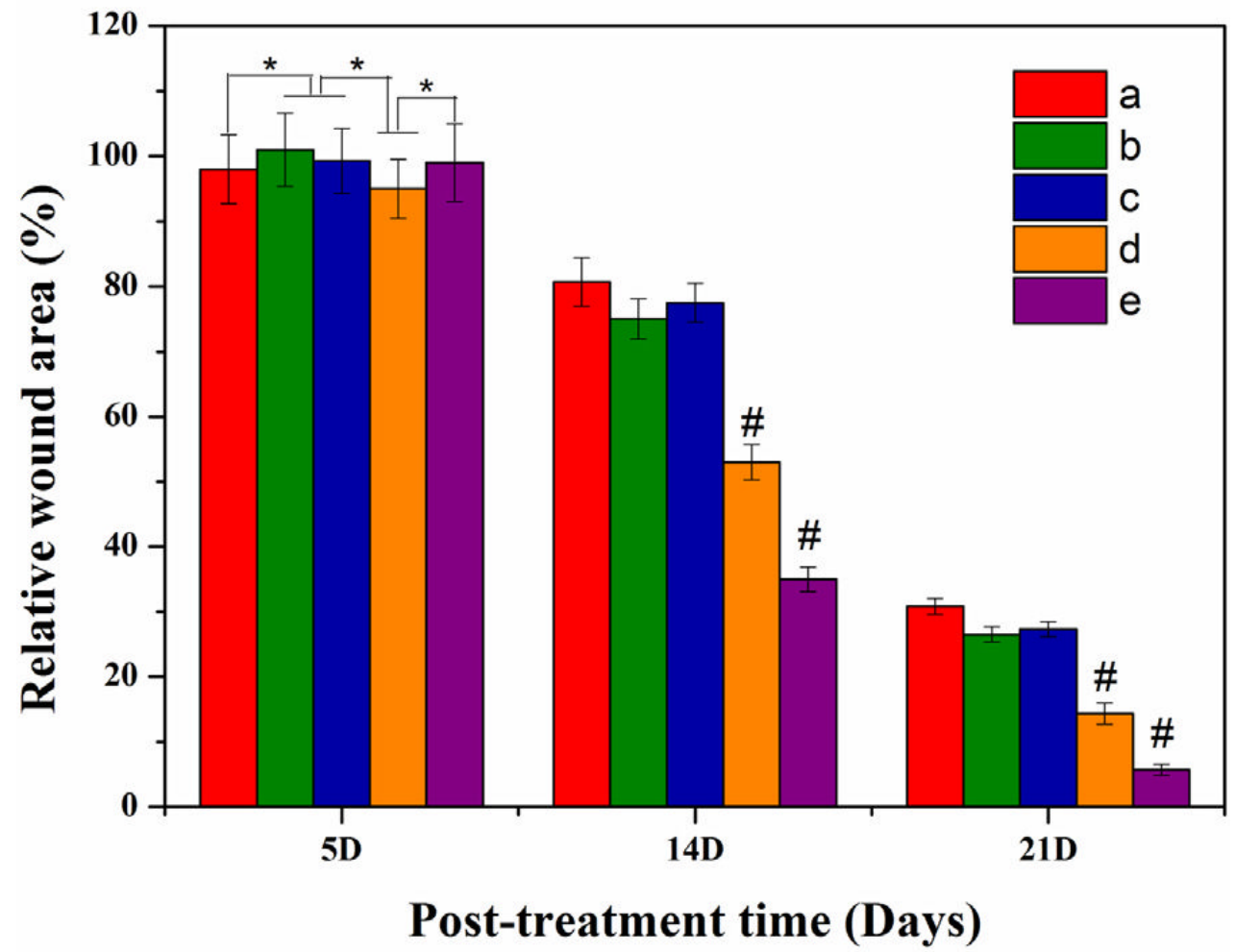

Figure 8. Relative wound areas 0, 5, 14 and 21 days post-operation. Results are from 7 rats, and expressed as mean \pm S.D. The wounds were treated with (a) a commercial 875 gauze alone; (b) the same type of gauze soaked with a $0.9 \% \mathrm{CIF}$ solution; (c) CIF solution; (d) S8; and, (e) S6. * indicates a significant difference between two groups $(\mathrm{p}<0.05)$ and \# a significant difference to all other groups at the same timepoint $(\mathrm{p}<$ $0.05)$. 


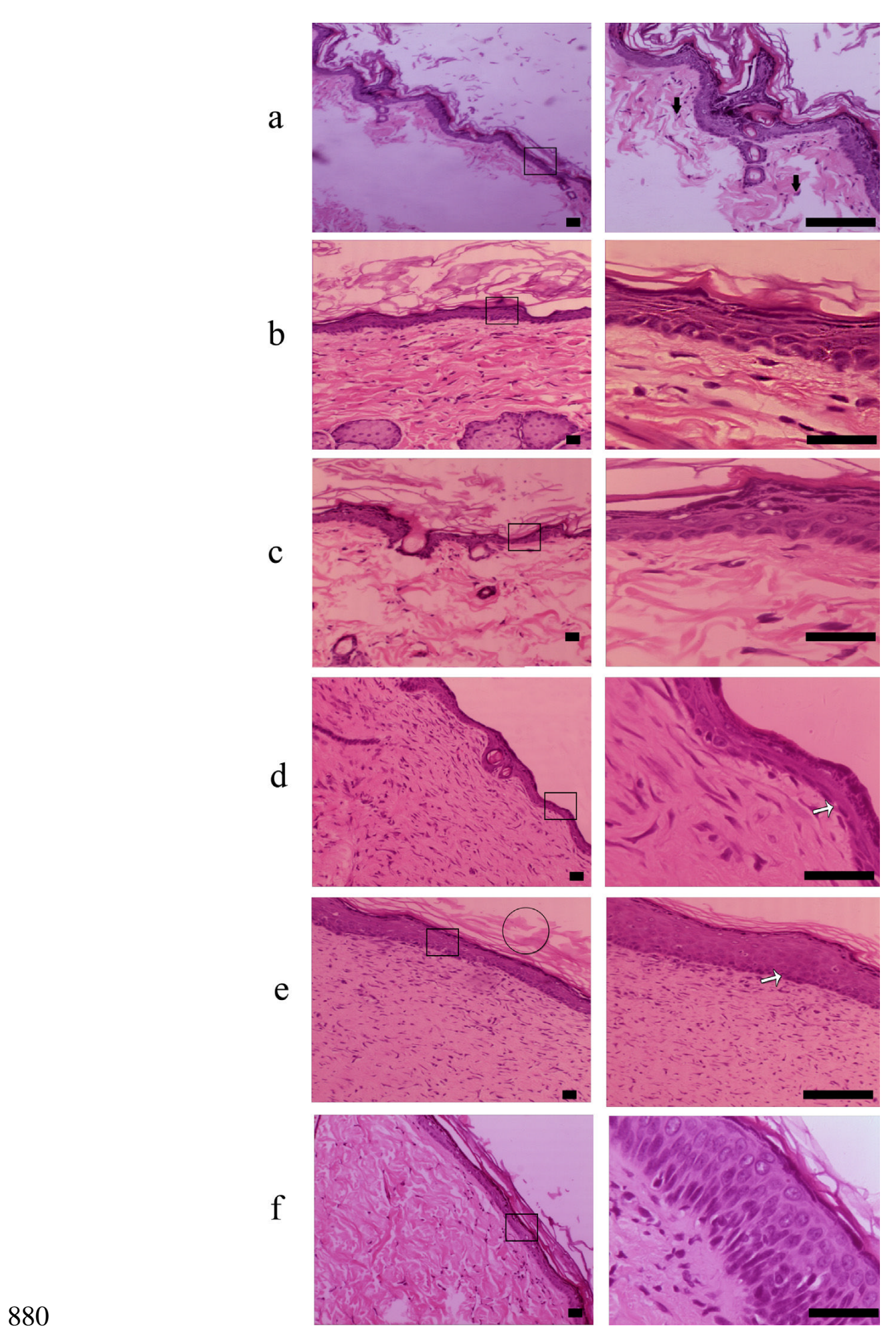

Figure 9. HE staining for the epithelialization of skin wounds treated for 21 days with (a) a commercial gauze alone; (b) the same type of gauze soaked with a $0.9 \% \mathrm{CIF}$ solution; (c) CIF solution; (d) S8; and, (e) S6, and are presented together with data for (f) normal skin without any wounds or treatment. These are representative images

885 from three rats of each group, and scale bars represent $40 \mathrm{~mm}$. Images on the right have larger magnificent than those on the left. 

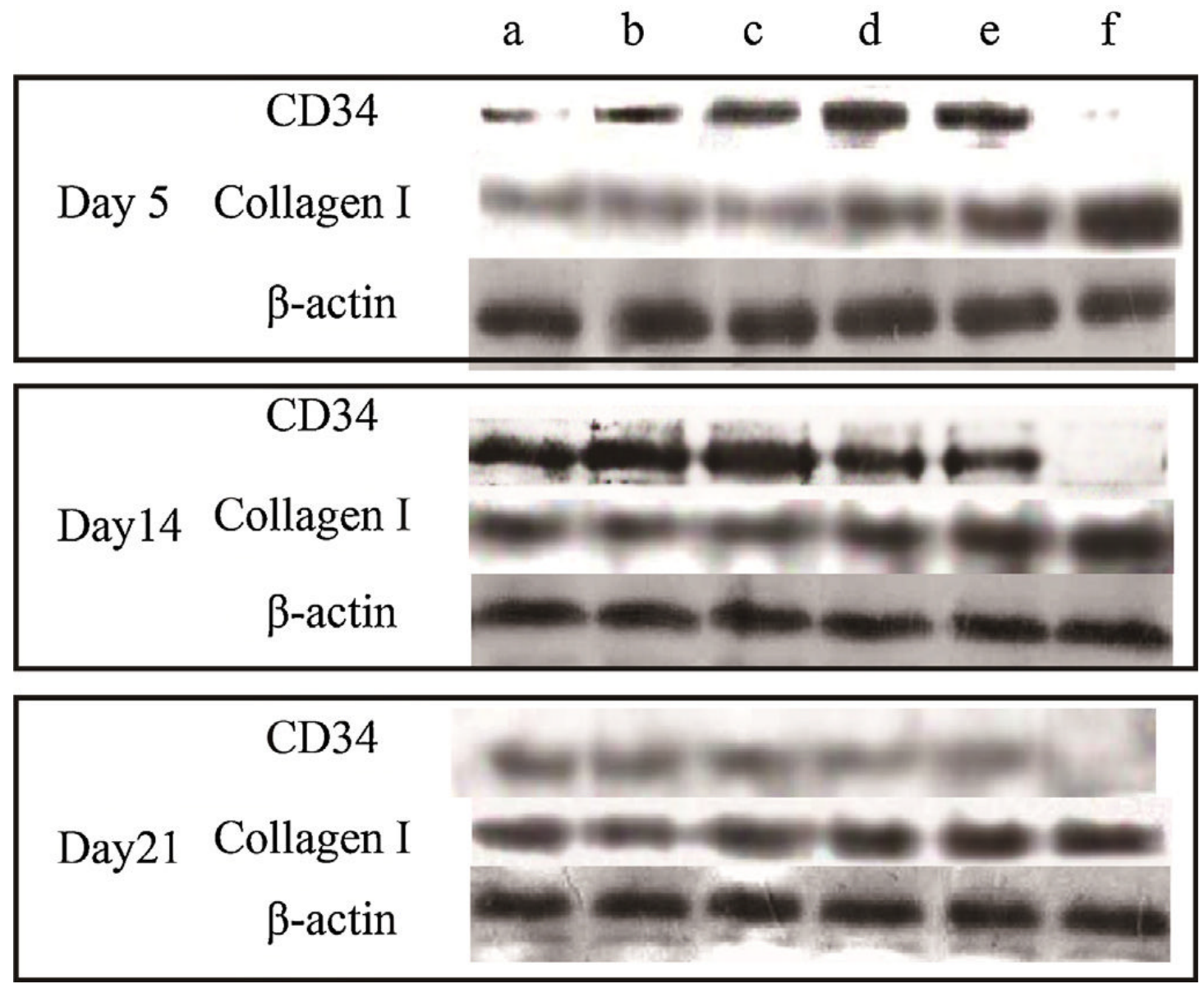

Figure 10. Western blotting results for CD34 and collagen expression in skin wounds treated with (a) gauze; (b) gauze soaked with a $0.9 \%$ CIF solution; (c) CIF solution; (d) S8; (e) S6; and, (f) normal skin after 5, 14 and 21 days. Total proteins were prepared from skin wounds, and $\beta$-actin expression was used as the protein loading control. 